The Astrophysical Journal, 672:1013-1037, 2008 January 10

(C) 2008. The American Astronomical Society. All rights reserved. Printed in U.S.A.

\title{
THE SPITZER c2d SURVEY OF LARGE, NEARBY, INTERSTELLAR CLOUDS. VII. OPHIUCHUS OBSERVED WITH MIPS
}

\author{
Deborah L. Padgett, ${ }^{1}$ Luisa M. Rebull, ${ }^{1}$ Karl R. Stapelfeldt,${ }^{2}$ Nicholas L. Chapman, ${ }^{3}$ Shih-Ping Lai, ${ }^{3,4,5}$ \\ Lee G. Mundy, ${ }^{3}$ Neal J. Evans II ${ }^{6}$ Timothy Y. Brooke, ${ }^{1}$ Lucas A. Cieza, ${ }^{6}$ William J. Spiesman, ${ }^{6}$ \\ Alberto Noriega-Crespo, ${ }^{1}$ Caer-Eve McCabe, ${ }^{1,2}$ Lori E. Allen, ${ }^{7}$ Geoffrey A. Blake, ${ }^{8}$ \\ Paul M. Harvey, ${ }^{6}$ Tracy L. Huard, ${ }^{7}$ Jes K. Jørgensen, ${ }^{7,9}$ David W. Koerner, ${ }^{10}$ \\ Philip C. Myers, ${ }^{7}$ Annelia I. Sargent, ${ }^{11}$ Peter Teuben, ${ }^{3}$ Ewine F. van Dishoeck, ${ }^{12}$ \\ ZaHed WaHhaJ, ${ }^{10,13}$ and KaISA E. YounG ${ }^{6,14}$ \\ Received 2007 January 10; accepted 2007 September 18
}

\begin{abstract}
We present maps of $14.4 \mathrm{deg}^{2}$ of the Ophiuchus dark clouds observed by the Spitzer Space Telescope Multiband Imaging Photometer for Spitzer (MIPS). These high-quality maps depict both numerous point sources and extended dust emission within the star-forming and non-star-forming portions of these clouds. Using PSF-fitting photometry, we detect 5779 sources at $24 \mu \mathrm{m}$ and 81 sources at $70 \mu \mathrm{m}$ at the $10 \sigma$ level of significance. Three hundred twenty-three candidate young stellar objects (YSOs) were identified according to their positions on the MIPS/2MASS $K$ versus $K-[24]$ color-magnitude diagrams, as compared to $24 \mu \mathrm{m}$ detections in the SWIRE extragalactic survey. We find that more than half of the YSO candidates, and almost all those with protostellar Class I spectral energy distributions, are confined to the known cluster and aggregates.
\end{abstract}

Subject headings: infrared: stars — ISM: clouds — stars: formation

\section{INTRODUCTION}

The Ophiuchus molecular cloud complex is one of the most well-known regions of nearby active star formation. For the Lynds 1688 cloud alone, SIMBAD lists 770 references as of summer 2006. The clouds $\left(\alpha=16^{\mathrm{h}} 28^{\mathrm{m}} 06^{\mathrm{s}}, \delta=-24^{\circ} 32^{\prime} 30^{\prime \prime}[\mathrm{J} 2000.0]\right)$ are considered to be at a distance of $125 \pm 25 \mathrm{pc}$ (de Geus et al. 1989), although more distant estimates are seen in the literature (145 pc, de Zeeuw et al. 1999; 160 pc, Chini 1981). The stellar population of the Ophiuchus clouds has been the subject of many imaging surveys (Grasdalen et al. 1973; Vrba et al. 1975; Wilking \& Lada 1983; Lada \& Wilking 1984; Rieke et al. 1989; Strom et al. 1995; Barsony et al. 1989, 1997, 2005; Greene \& Young 1992; Comeron et al. 1993; Bontemps et al. 1998; Allen

\footnotetext{
1 Spitzer Science Center, MC 220-6, California Institute of Technology, Pasadena, CA 91125; dlp@ipac.caltech.edu.

2 Jet Propulsion Laboratory, MS 183-900, 4800 Oak Grove Drive, Pasadena, CA 91109.

3 Department of Astronomy, University of Maryland, College Park, MD 20742.

4 Institute of Astronomy and Department of Physics, National Tsing Hua University, Hsinchu 30043, Taiwan.

5 Academia Sinica Institute of Astronomy and Astrophysics, P.O. Box 23-141, Taipei 106, Taiwan.

6 Department of Astronomy, University of Texas at Austin, 1 University Station C1400, Austin, TX 78712.

${ }^{7}$ Harvard-Smithsonian Center for Astrophysics, 60 Garden Street, MS 42, Cambridge, MA 02138.

${ }^{8}$ Division of Geological and Planetary Sciences, California Institute of Technology, MS 150-21, Pasadena, CA 91125.

9 Current address: Argelander-Institut für Astronomie, Auf dem Hügel 71, D-53121 Bonn, Germany.

10 Department of Physics and Astronomy, Northern Arizona University, NAU Box 6010, Flagstaff, AZ 86011-6010.

11 Division of Physics, Mathematics, and Astronomy, California Institute of Technology, MS 105-24, Pasadena, CA 91125.

12 Leiden Observatory, Leiden University, P.O. Box 9513, NL 2300 RA Leiden, The Netherlands.

${ }^{13}$ Current address: Institute for Astronomy, University of Hawaii, 2680 Woodlawn Drive, Honolulu HI 96822-1897.

14 Current address: Department of Physical Sciences, Nicholls State University, Thibodaux, LA 70301.
}

et al. 2002; Duchêne et al. 2004; etc.) and spectroscopic surveys (Bouvier \& Appenzeller 1992; Greene \& Meyer 1995; Martín et al. 1998; Wilking et al. 1999, 2005; Luhman \& Rieke 1999; Cushing et al. 2000; Natta et al. 2006; etc.). Many thousands of sources have been cataloged at near-IR wavelengths; however, the majority of these sources are extincted background stars (Barsony et al. 1997).

L1688 contains the most famous star-forming cluster in this region, with of order 100 stars $<1$ Myr old, including several extremely young Class 0-I sources (Bontemps et al. 2001; Duchêne et al. 2004; Haisch et al. 2002; and many older studies). The vicinity of this cluster is covered in bright and dark nebulosity, and Herbig-Haro flows criss-cross the entire region (Gomez et al. 1998; Phelps \& Barsony 2004). Most of the known young stellar objects (YSOs) are found within an embedded cluster only a few parsecs in extent, making Ophiuchus one of the higher density environments among the nearby low-mass star formation regions (Grasdalen et al. 1973; Wilking \& Lada 1983, 1989; etc.). It is also a region forming intermediate-mass stars, bridging the gap between Taurus (a region of low-mass, isolated star formation) and Orion (a dense cluster with massive O stars). In Ophiuchus, the principal region of low-mass star formation is in the vicinity of several intermediate- to high-mass stars of the Sco OB1 association (de Zeeuw et al. 1999), and a number of A and B stars appear to be embedded within the cloud (Klose 1986; Devito \& Hayward 1998).

The molecular material in the Lynds 1688 and 1689 clouds extends far beyond the boundaries of the extant surveys for YSOs. To the southeast of L1688 is the L1689 cloud, which extends to the east in the L1712 and L1729 streamers. Only a few isolated YSOs are known in this fairly massive cloud, leading Nutter et al. (2006) to dub it "the dog that didn't bark." Their submillimeter continuum mapping survey indicates that most of the mass of L1689 resides in distributed cloud material rather than dense cores, unlike L1688, and suggests that the proximity of L1688 to $\sigma$ Sco explains its much greater star formation efficiency. Filamentary clouds (L1709, L1740, and L1744+L1755+L1765 = "Oph North") also extend to the northeast of L1688. To date, 
TABLE 1

Summary of Observations (Program 177)

\begin{tabular}{|c|c|c|c|c|}
\hline \multirow[b]{2}{*}{ FIELD } & \multicolumn{2}{|c|}{ Map Center } & \multirow[b]{2}{*}{ FIRST-EPOCH AORKEY } & \multirow[b]{2}{*}{ SECOND-EPOCH AORKEY } \\
\hline & $\alpha(\mathrm{J} 2000.0)$ & $\delta(\mathrm{J} 2000.0)$ & & \\
\hline Ophi-mips1................. & 164550.0 & -212116.0 & 5745408 & 5747200 \\
\hline Ophi-mips $2 \ldots \ldots \ldots \ldots \ldots . . .$. & 164324.0 & -215738.0 & 5745664 & 5747456 \\
\hline 1689-mips1................. & 164316.0 & -240909.4 & 5748992 & 5753344 \\
\hline $1689-\operatorname{mips} 2 \ldots \ldots \ldots \ldots . .$. & 163827.0 & -241802.0 & 5749248 & 5753600 \\
\hline $1689-\operatorname{mips} 3 \ldots \ldots \ldots \ldots \ldots$ & 163512.0 & -243400.0 & 5749504 & 5753856 \\
\hline 1688-mips1.................. & 163333.0 & -242042.0 & 5757952 & 5766144 \\
\hline 1688 -mips $2 \ldots \ldots \ldots \ldots \ldots$ & 163301.5 & -231820.0 & 5758208 & 5766400 \\
\hline 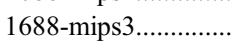 & 163159.2 & -245753.0 & 5758464 & 5766656 \\
\hline 1688 -mips $4 \ldots \ldots \ldots \ldots \ldots$ & 163100.0 & -233750.1 & 5758720 & 5766912 \\
\hline 1688 -mips $5 \ldots \ldots \ldots \ldots \ldots . . . .$. & 163003.0 & -250822.0 & 6605568 & 6605824 \\
\hline 1688 -mips6................... & 162756.5 & -241112.0 & 5759488 & 5767680 \\
\hline 1688-mips $7 \ldots \ldots \ldots \ldots \ldots . . . .$. & 162546.0 & -241229.0 & 5759744 & 5676936 \\
\hline 1688 -mips $8 \ldots \ldots \ldots \ldots \ldots$ & 162409.0 & -235003.0 & 5760000 & 5768192 \\
\hline 1688 -mips $9 \ldots \ldots \ldots \ldots \ldots$ & 162152.5 & -234345.0 & 5760256 & 5768448 \\
\hline 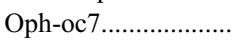 & 162352.0 & -215738.0 & 5778432 & 5778688 \\
\hline 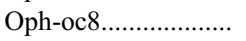 & 162312.0 & -262849.0 & 5778944 & 5779200 \\
\hline
\end{tabular}

NoTE.-Units of right ascension are hours, minutes, and seconds, and units of declination are degrees, arcminutes, and arcseconds.

these clouds are lacking in high-resolution mid-IR surveys to identify and characterize their YSO content. Only the IRAS survey and the Two Micron All Sky Survey (2MASS) exist for these regions. The ISOCAM survey of Oph covered only $0.7 \mathrm{deg}^{2}$ (Bontemps et al. 1998). Those authors claim that most of the YSOs outside of this central region are weak-line and post-T Tauri stars, and indeed, this is generally true based on only the IRAS and ROSAT studies of the region. The current study using the Spitzer Space Telescope is capable of detecting low-luminosity Class 0 -III infrared sources throughout a much larger surveyed area.

The Ophiuchus molecular cloud complex is one of five starforming regions observed with Spitzer as a part of the "Molecular Cores to Planet-forming Disks" (c2d) Legacy project (Evans et al. 2003). The goal of this survey is to characterize the circumstellar material of young stars and substellar objects in these clouds. This paper is one in a series which present the c2d largecloud mapping results made with Spitzer's Infrared Camera (IRAC) and Multiband Imaging Photometer for Spitzer (MIPS). Others in the series report results for Chamaeleon II (MIPS, Young et al. 2005; IRAC, Porras et al. 2007), Lupus (MIPS, Chapman et al. 2007), Perseus (MIPS, Rebull et al. 2007; IRAC, Jørgensen et al. 2006), and Serpens (IRAC, Harvey et al. 2006; MIPS, Harvey et al. 2007). These papers endeavor to present the results for each cloud and instrument in a standard format. Although the current work principally discusses the Ophiuchus MIPS maps, data from IRAC are also integrated into sections regarding source classification and individual objects. The IRAC results for Ophiuchus will be presented in L. E. Allen et al. 2008, in preparation.

The c2d MIPS survey of Ophiuchus attempts to determine the importance of a more distributed mode of star formation in the less-studied areas outside the Oph core by covering more than $13.8 \mathrm{deg}^{2}$ in the region where $A_{V} \geq 5$ at sensitivity levels considerably better than that of ISOCAM at mid-IR wavelengths and many orders of magnitude better than IRAS and ISOPHOT at far-IR wavelengths. The three MIPS filters at 24, 70, and $160 \mu \mathrm{m}$ are ideally suited for linking the circumstellar environment of individual stars to the interstellar environment of the cloud. The MIPS $24 \mu \mathrm{m}$ band is especially useful for identifying stars with infrared excess, since stellar photospheric emission has decreased by 2 orders of magnitude from the blackbody peak, increasing the contrast with peak emission from cool dust. Thus, stars with warm young disks are generally extremely bright at $24 \mu \mathrm{m}$ relative to stars without disk emission. MIPS $70 \mu \mathrm{m}$ emission is also seen in the brighter envelopes and disks and in interstellar regions where dust is heated by young stars. MIPS $160 \mu \mathrm{m}$ traces cool dust in both circumstellar and interstellar regions, similar to the IRAS $100 \mu \mathrm{m}$ band. The combination of MIPS data with surveys at other wavelengths enables detailed studies of YSO spectral energy distributions (SEDs) and the cloud environment. The most recent millimeter continuum maps of this region have been presented by Young et al. (2006), who used the Caltech Submillineter Observatory (CSO) Bolocam to map the region observed by Spitzer. High-quality millimeter molecular-line maps of Ophiuchus also covering the Spitzer survey are available from the COMPLETE team (Ridge et al. 2006).

In this paper we first present the Spitzer MIPS observations of Ophiuchus ( $(2)$. Following the observations section, we discuss results, including multiwavelength images (§ 3.1), MIPS source counts $(\S 3.2)$, band-merging with IRAC and 2MASS (§ 3.3), and a variety of color-color and color-magnitude diagrams ( $(3.4)$. Then we investigate recovery of IRAS objects $(\S 3.5)$ and variability at $24 \mu \mathrm{m}$ on timescales of $3-8 \mathrm{hr}$ in $\S 3.6$. We highlight the SEDs and imaging of several individual objects in $\S 4.1$. We then discuss the distribution of infrared excess sources in Ophiuchus and comment on the degree of clustering found throughout the region (§4.2). Finally, we summarize our main points in $\S 5$.

\section{OBSERVATIONS AND DATA REDUCTION}

\subsection{MIPS}

The MIPS observations of Ophiuchus were conducted on 2004 March 18-19 and covered $13.8 \mathrm{deg}^{2}$. Two off-cloud fields totalling $0.6 \mathrm{deg}^{2}$ were observed with the intention of characterizing the background star counts. The center of this large map is roughly at $(\alpha, \delta)(\mathrm{J} 2000.0)=\left(16^{\mathrm{h}} 33^{\mathrm{m}} 18.0^{\mathrm{s}},-24^{\circ} 15^{\prime}\right)$, or $(l, b)=$ $\left(354^{\circ},+16.0^{\circ}\right)$, or ecliptic coordinates $\left(251^{\circ},-2.5^{\circ}\right)(\mathrm{J} 2000.0)$. The area mapped with MIPS completely covers the c2d IRAC 


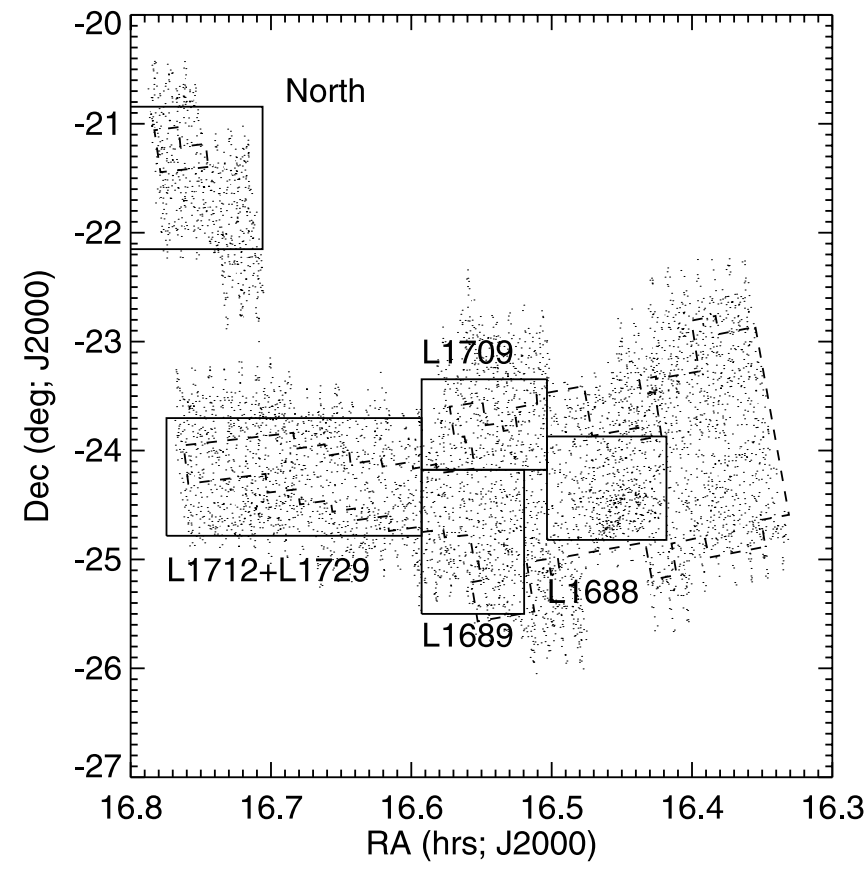

FIG. 1.-Location of MIPS coverage (points are MIPS-24 detections), with the region of IRAC coverage (dashed line) indicated. The smaller squares (solid line) indicates the regions defined to be L1688, L1689, and Oph North.

map regions in Ophiuchus, which included all the cloud area above the $A_{V}=5$ contour. The combination of the cloud being extended perpendicular to the MIPS scan direction, and the fixed scan length options available for use with MIPS, dictated that the area covered by the Ophiuchus MIPS maps be more than twice the area mapped with IRAC.

These observations were part of Spitzer program 177; the AORKEYs appear in Table 1. Fast-scan maps were obtained at two separate epochs separated by a few hours. At each epoch, the spacing between adjacent scan legs was $240^{\prime \prime}$. The second-epoch observation was offset $125^{\prime \prime}$ in the cross-scan direction from the first to fill in the $70 \mu \mathrm{m}$ sky coverage, which was incomplete at each individual epoch. Furthermore, the second-epoch scan was also offset $80^{\prime \prime}$ from the first in the scan direction to maximize $160 \mu \mathrm{m}$ map coverage. These mapping parameters resulted in every part of the map being imaged at two epochs at $24 \mu \mathrm{m}$ and only one epoch at 70 and $160 \mu \mathrm{m}$, with total integration times of 30,15 , and $3 \mathrm{~s}$ at each point in the map (respectively). Even with this strategy, the $160 \mu \mathrm{m}$ maps have some gaps, which makes point-source photometry especially uncertain at this wavelength. In addition, the $160 \mu \mathrm{m}$ array is saturated in the region at the center of the L1688 cloud.

Figure 1 shows the regions of three-band coverage with MIPS and of four-band coverage with IRAC. In addition to the c2d data, smaller MIPS maps of Ophiuchus were obtained by Spitzer Guaranteed Time Observers. To maintain uniform sensitivity in our maps, these data were not incorporated into the current study.

The observations were conducted at two separate epochs separated by about $3-8 \mathrm{hr}$ to permit asteroid removal in this low ecliptic latitude $\left(\sim 0^{\circ}\right)$ field. Study of the asteroid population in Taurus at a similar ecliptic latitude $\left(0^{\circ}-7^{\circ}\right)$ indicates there are roughly 250 asteroids $\mathrm{deg}^{-2}$ with $24 \mu \mathrm{m}$ flux densities $\geq 1.6 \mathrm{mJy}$ (Stapelfeldt et al. 2006). By extracting sources separately from the two epochs and comparing the source lists, transient sources were eliminated from our MIPS catalog. This observation technique also enables a study of $24 \mu \mathrm{m}$ source variability on a timescale of several hours (see $\S 3.6$ ).
TABLE 2

Box Regions Used to Define Individual Clouds

\begin{tabular}{|c|c|c|c|c|}
\hline \multirow[b]{2}{*}{ Cluster } & \multicolumn{2}{|c|}{ SoutheAst Box Corner } & \multicolumn{2}{|c|}{ NorthWEst Box CORner } \\
\hline & $\alpha(\mathrm{J} 2000.0)$ & $\delta(\mathrm{J} 2000.0)$ & $\alpha(\mathrm{J} 2000.0)$ & $\delta(\mathrm{J} 2000.0)$ \\
\hline L1688.. & 163012.0 & -244915.6 & 162505.5 & -234915.6 \\
\hline L1689 ........ & 163533.6 & -253000.0 & 163110.6 & $\begin{array}{llll}-24 & 10 & 37.2\end{array}$ \\
\hline L1709 ……........... & 163533.6 & $-24 \quad 1037.2$ & 163012.0 & -232045.6 \\
\hline $\mathrm{L} 1712+\mathrm{L} 1729 \ldots .$. & 164628.1 & -244658.8 & 163533.6 & $\begin{array}{llll}-23 & 42 & 07.2\end{array}$ \\
\hline North ........................ & $1648 \quad 10.3$ & -220903.6 & 164221.6 & -205034.8 \\
\hline
\end{tabular}

Note.- Units of right ascension are hours, minutes, and seconds, and units of declination are degrees, arcminutes, and arcseconds.

The MIPS observations of Ophiuchus included two small offcloud regions with total area of $0.66 \mathrm{deg}^{2}$, in addition to the $13.8 \mathrm{deg}^{2}$ of the main map. These were intended to provide statistics on the stellar and extragalactic backgrounds. However, these regions were found to be too small and sparse to yield reliable statistics. In addition, one of the fields inadvertently included the large globular cluster M4, which severely biases its source counts. Thus, we did not utilize these off-cloud regions; instead, we compare our statistics to the $6.15 \mathrm{deg}^{2}$ ELAIS N1 field mapped by the Spitzer Wide-Area Infrared Extragalactic ("SWIRE") survey (Shupe et al. 2008), which was rereduced and analyzed using the $\mathrm{c} 2 \mathrm{~d}$ pipeline. In the low ecliptic latitude Ophiuchus region, MIPS source counts are dominated by asteroids and the extragalactic background rather than stellar photospheres, and thus, a comparison to the SWIRE field is invaluable despite its considerably different Galactic latitude. However, we do include the offcloud fields (OC1 and OC2) in the table of statistics (see $\S 3.2$ ).

We started with the Spitzer Science Center (SSC)-pipelineproduced basic calibrated data (BCDs), version S11.4. Each MIPS channel was then processed differently, and therefore, each is discussed separately below. Additional detail on the data reduction process can be found in Young et al. (2005), Chapman et al. (2007), and Rebull et al. (2007).

Standard c2d pipeline processing was used for MIPS-24 (Young et al. 2005; Evans 2003). After some corrections for nonuniform readout variations (jailbars) were applied to individual frames (BCDs), these data were transformed into single-epoch mosaics using the SSC MOPEX software (following Chapman et al. 2007). Source lists were extracted using c2dphot (Harvey et al. 2006) to measure flux densities using point-spread function fitting. The final working 2005 catalog as assembled consists of all detections at MIPS-24 with high-quality c2d catalog detection flags (Evans et al. 2007, the c2d Data Delivery document ${ }^{15}$ ) and detections at both epochs. While resulting in a shallower survey than would be possible using other combinations of flags, this ensures that no asteroids are included in the final catalog. The zero point used to convert these flux densities to magnitudes was 7.14 Jy, based on extrapolation from the Vega spectrum as published in the MIPS Data Handbook. There were 5779 total point sources detected at $24 \mu \mathrm{m}$ meeting our criteria, ranging from 0.672 to $3910 \mathrm{mJy}$. Note that the spatial resolution of MIPS-24 is $\sim 6^{\prime \prime}$, with a $2.55^{\prime \prime}$ pixel size.

For the MIPS-70 data we started with the automated pipelineproduced BCDs, both the filtered and unfiltered products. Then individual $\mathrm{BCDs}$ were mosaicked into one filtered and one unfiltered mosaic using the SSC software MOPEX (Makovoz

\footnotetext{
${ }^{15}$ See http://data.spitzer.caltech.edu/popular/c2d/20071101_enhanced_v1/ Documents/c2d_del_document.pdf.
} 


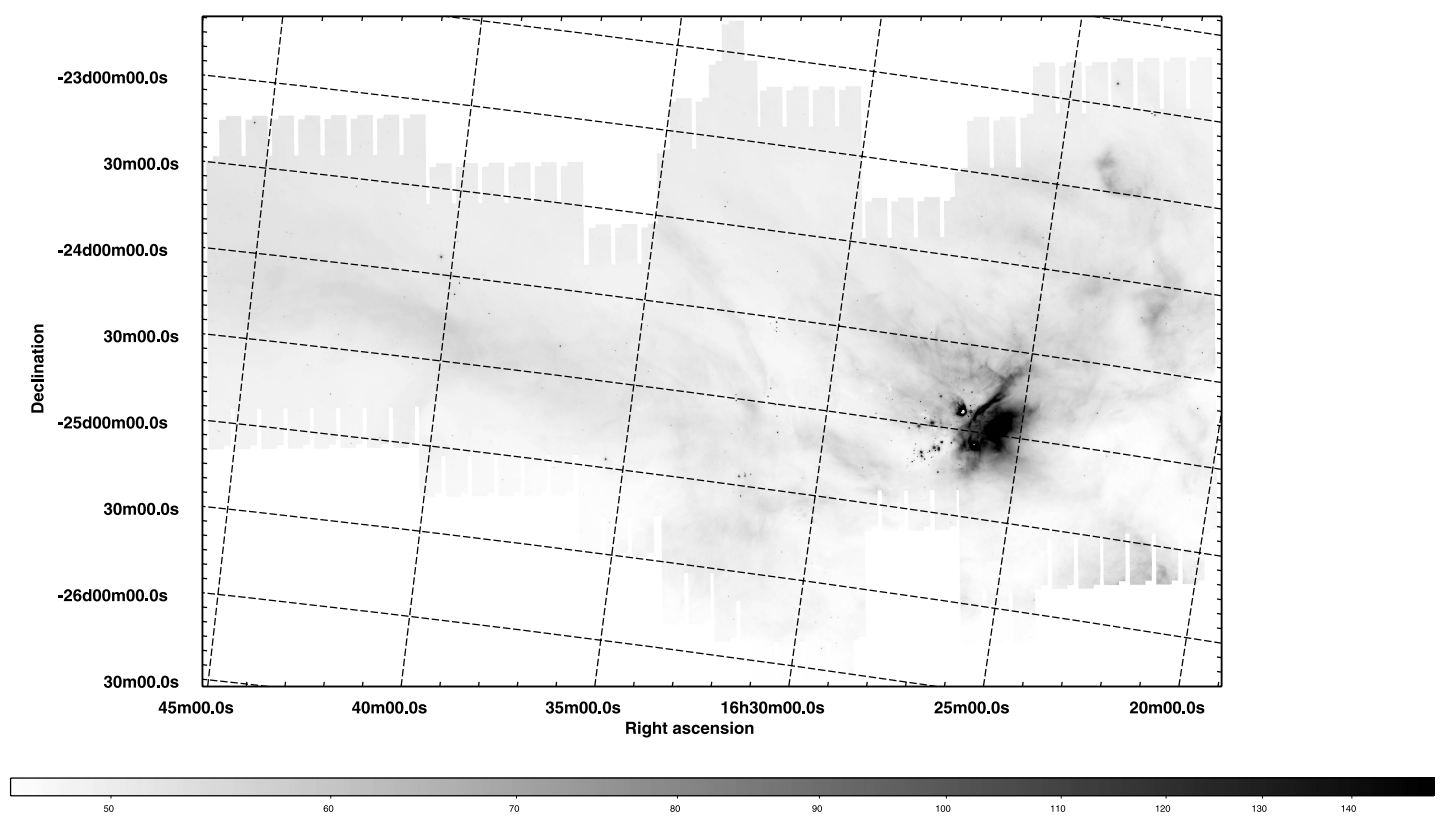

FIG. 2.- Spitzer MIPS $24 \mu \mathrm{m}$ mosaic of Ophiuchus clouds.

et al. 2006). We performed source detection and extraction using MOPEX/APEX on the entire mosaic. The source list was cleaned for instrumental artifacts via manual inspection of the $70 \mu \mathrm{m}$ image and comparison to the $24 \mu \mathrm{m}$ image; e.g., if there was some question as to whether a faint object seen at $70 \mu \mathrm{m}$ was real or an instrumental artifact, and comparison to the $24 \mu \mathrm{m}$ image revealed a $24 \mu \mathrm{m}$ source, then the $70 \mu \mathrm{m}$ object was judged to be a real source. About $80 \%$ of the $70 \mu \mathrm{m}$ objects had identifiable counterparts at $24 \mu \mathrm{m}$. The zero point used to convert the flux densities to magnitudes was $0.775 \mathrm{Jy}$, based on extrapolation from the Vega spectrum as published in the MIPS Data
Handbook. There were 81 total point sources detected at $70 \mu \mathrm{m}$. There were 19 objects brighter than $4 \mathrm{Jy}$, for which we employed aperture photometry with a $32^{\prime \prime}$ aperture and a $17 \%$ aperture correction. Note that the spatial resolution of MIPS-70 is $\sim 18^{\prime \prime}$.

The $160 \mu \mathrm{m}$ MIPS band presented challenges for point-source extraction due to scattered gaps in the map coverage. A small amount of smoothing was applied to the data in order to fill gaps. Then APEX was used to find sources throughout the image. Because of the large saturated region in L1688 and the highly structured nature of the bright diffuse emission, few isolated point sources were identified in our study. The zero point used to

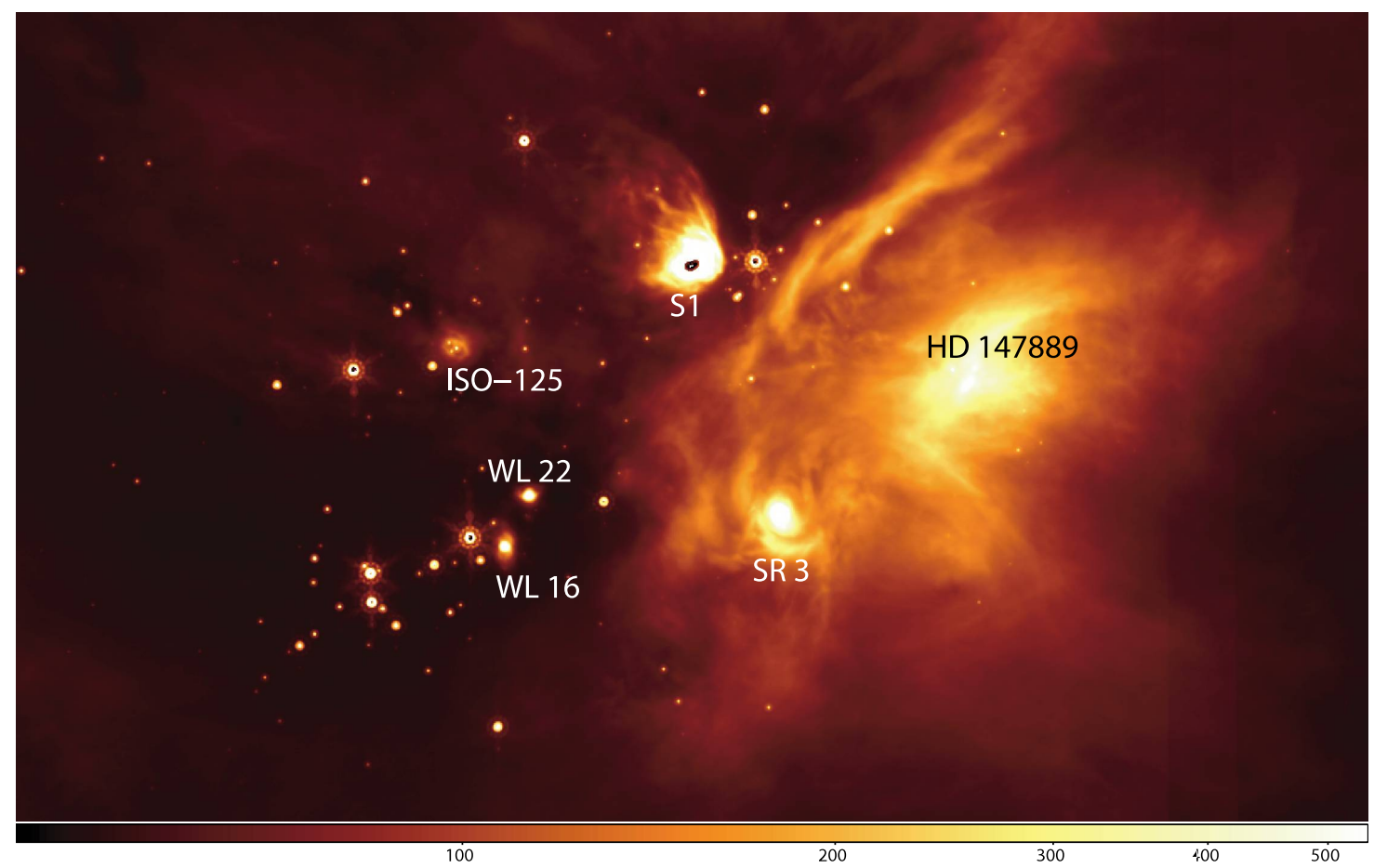

FIG. 3. - MIPS $24 \mu \mathrm{m}$ image: central $1^{\circ} \times 0.5^{\circ}$ region of Lynds 1688 . 


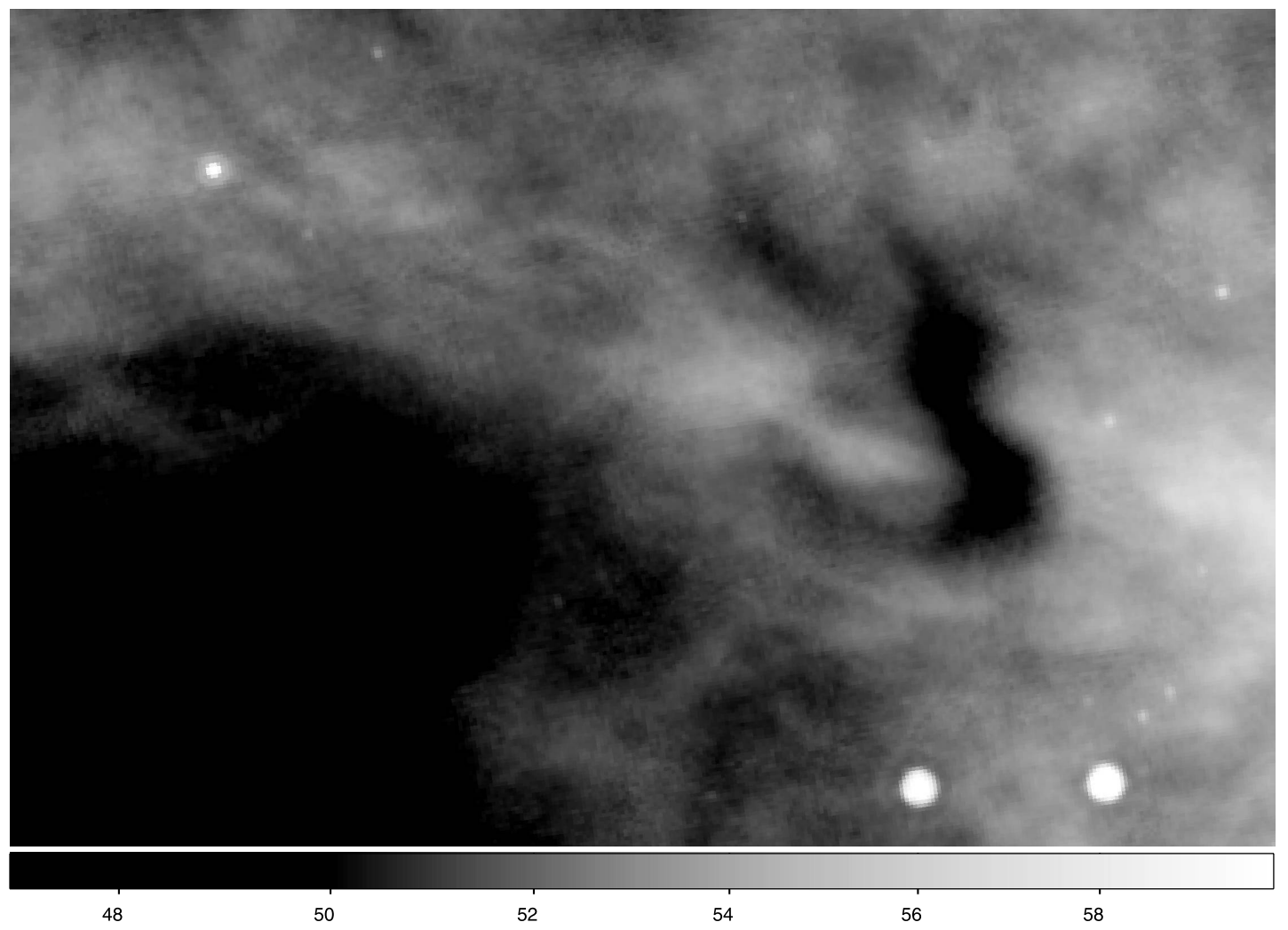

FIG. 4.-Dark clouds at $24 \mu \mathrm{m}$ found near $16^{\mathrm{h}} 28^{\mathrm{m}} 31^{\mathrm{s}},-24^{\circ} 18^{\prime} 40^{\prime \prime}$. The image spans $15^{\prime} \times 10^{\prime}$, with north up and east to the left.

convert the flux densities to magnitudes was $0.159 \mathrm{Jy}$, based on extrapolation from the Vega spectrum as published in the MIPS Data Handbook. There were eight total point sources detected at $160 \mu \mathrm{m}$, ranging from $876 \mathrm{mJy}$ to $19 \mathrm{Jy}$. About $60 \%$ of the $160 \mu \mathrm{m}$ objects had identifiable counterparts at 24 and $70 \mu \mathrm{m}$. The extreme brightness and saturation of the $160 \mu \mathrm{m}$ diffuse emission made detection of $160 \mu \mathrm{m}$ sources impossible in the most densely populated regions of the cluster. Finally, note that the spatial resolution of MIPS-160 is $\sim 36^{\prime \prime}$.
Although this paper is primarily about point sources, there is extensive extended emission in all three MIPS channels throughout the MIPS maps. The diffuse emission ranges in brightness from 50 to $570 \mathrm{MJy} \mathrm{sr}^{-1}$ at $24 \mu \mathrm{m}$, where the low value is set by the overall zodiacal emission in this low ecliptic latitude region. The $70 \mu \mathrm{m}$ extended emission ranges in surface brightness from $\sim 30$ to $5000 \mathrm{MJy} \mathrm{sr}^{-1}$, while the $160 \mu \mathrm{m}$ extended emission varies from 45 to $400 \mathrm{MJy} \mathrm{sr}^{-1}$ (with the central region of L1688 being saturated). This extreme background variation unfortunately

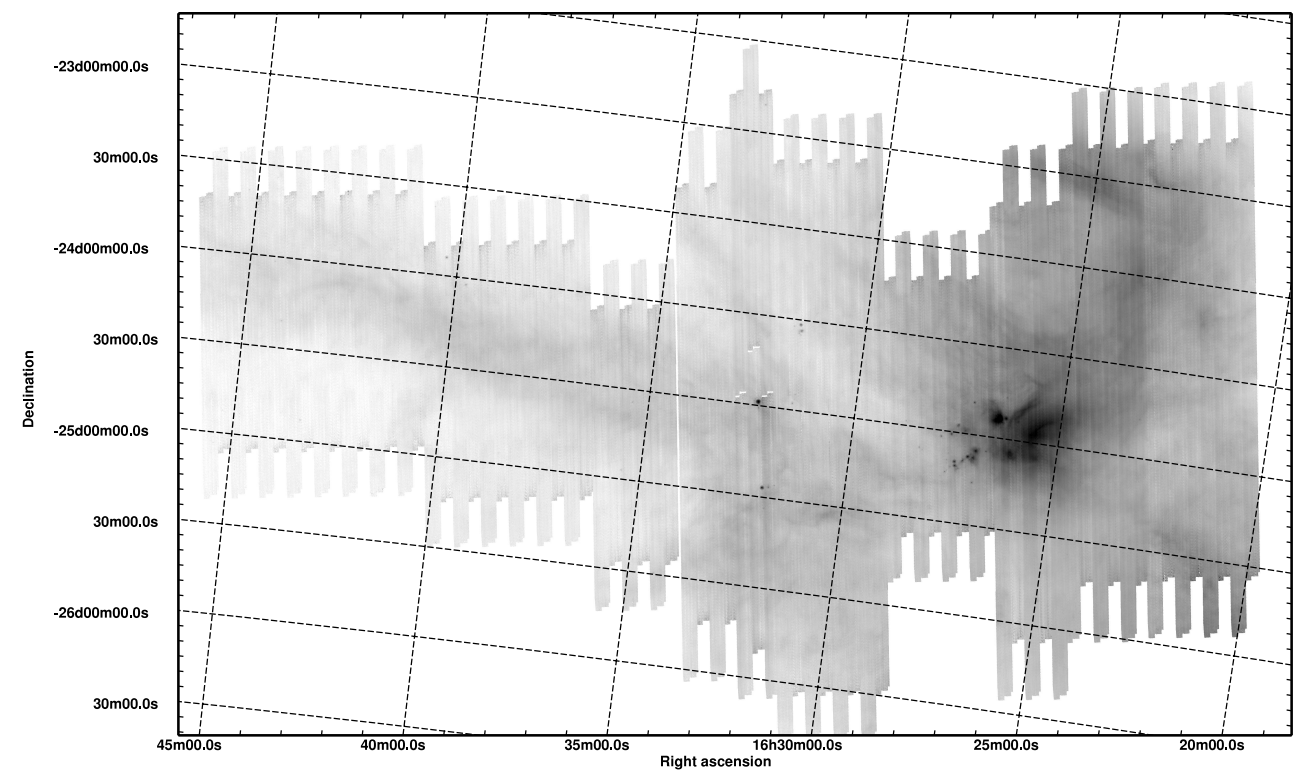

FIG. 5.-Spitzer MIPS $70 \mu \mathrm{m}$ mosaic of Ophiuchus clouds. 


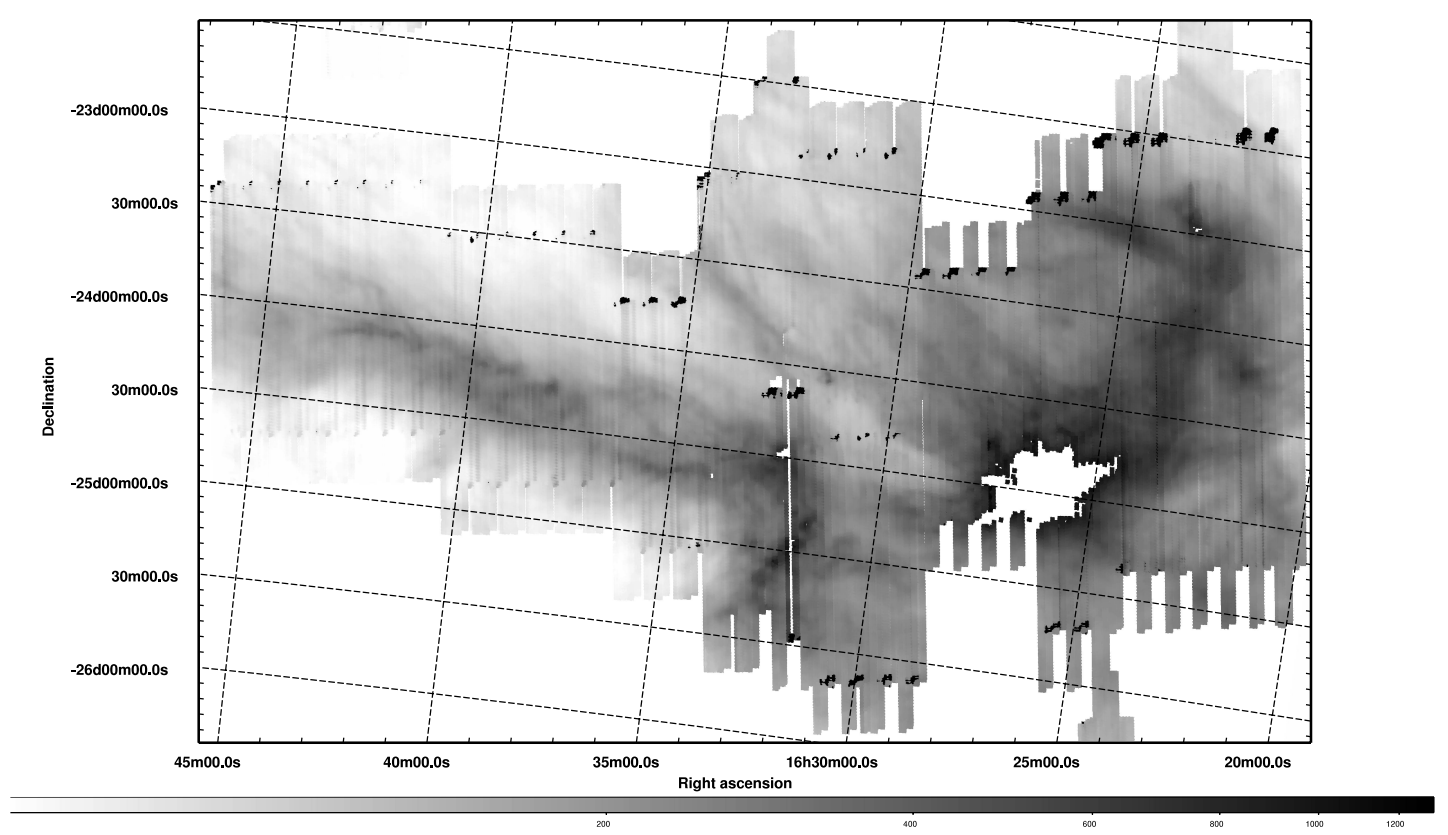

FIG. 6.-Spitzer MIPS $160 \mu$ m mosaic of Ophiuchus clouds. The central region of the cloud is saturated in our maps.

results in highly nonuniform point source detection sensitivity in our survey. Our region of worst sensitivity coincides with the central core of L1688, where the most intense past studies have been performed. Thus, our Spitzer c2d survey should be considered complementary to past Infrared Space Observatory (ISO) and ground-based IR studies in Oph.

Because a large cloud complex is encompassed by our maps and the population of the individual clouds is known to have substantially different characteristics, we have chosen to separate out the properties of stars in L1688, L1689, L1709, and the
L1712+L1729 filaments (see Fig. 1). In addition, we have separated out the properties of the disconnected northern filaments (L1744+L1755+L1765), which we identify as Oph North. The above regions were defined as the rectangles shown in Figure 1, with the corners given in Table 2.

\subsection{CSO Sharc II}

Several L1709 sources were observed at $350 \mu \mathrm{m}$, using Sharc II at the CSO on 2007 April 23, 25, and 26. Sharc II is a $12 \times 32$ bolometer array, with a pixel size of $4.85^{\prime \prime}$ and a field of view of

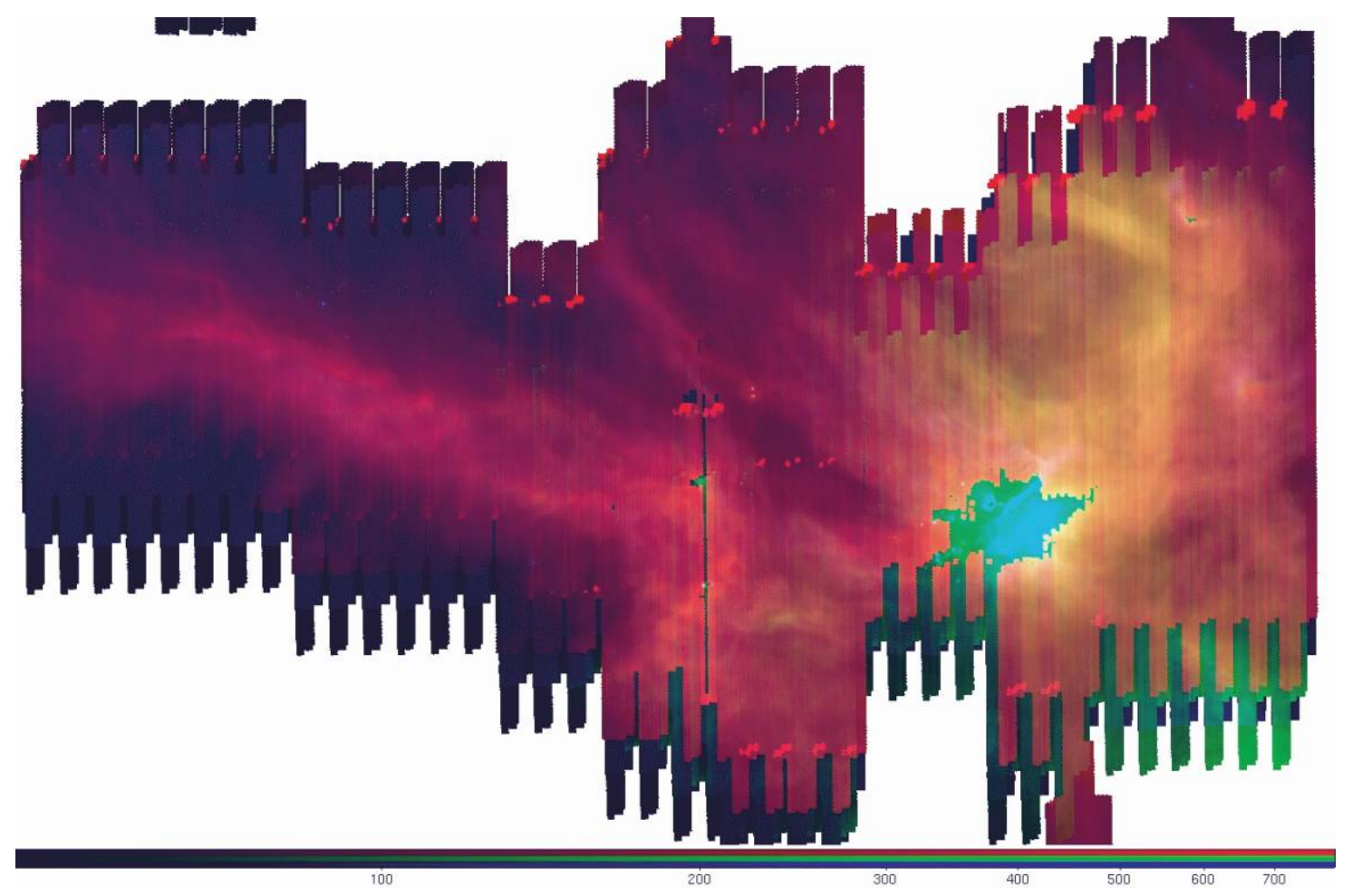

FIg. 7.-A 24 (blue), 70 (green), and $160 \mu \mathrm{m}$ (red) mosaic of Ophiuchus clouds. 


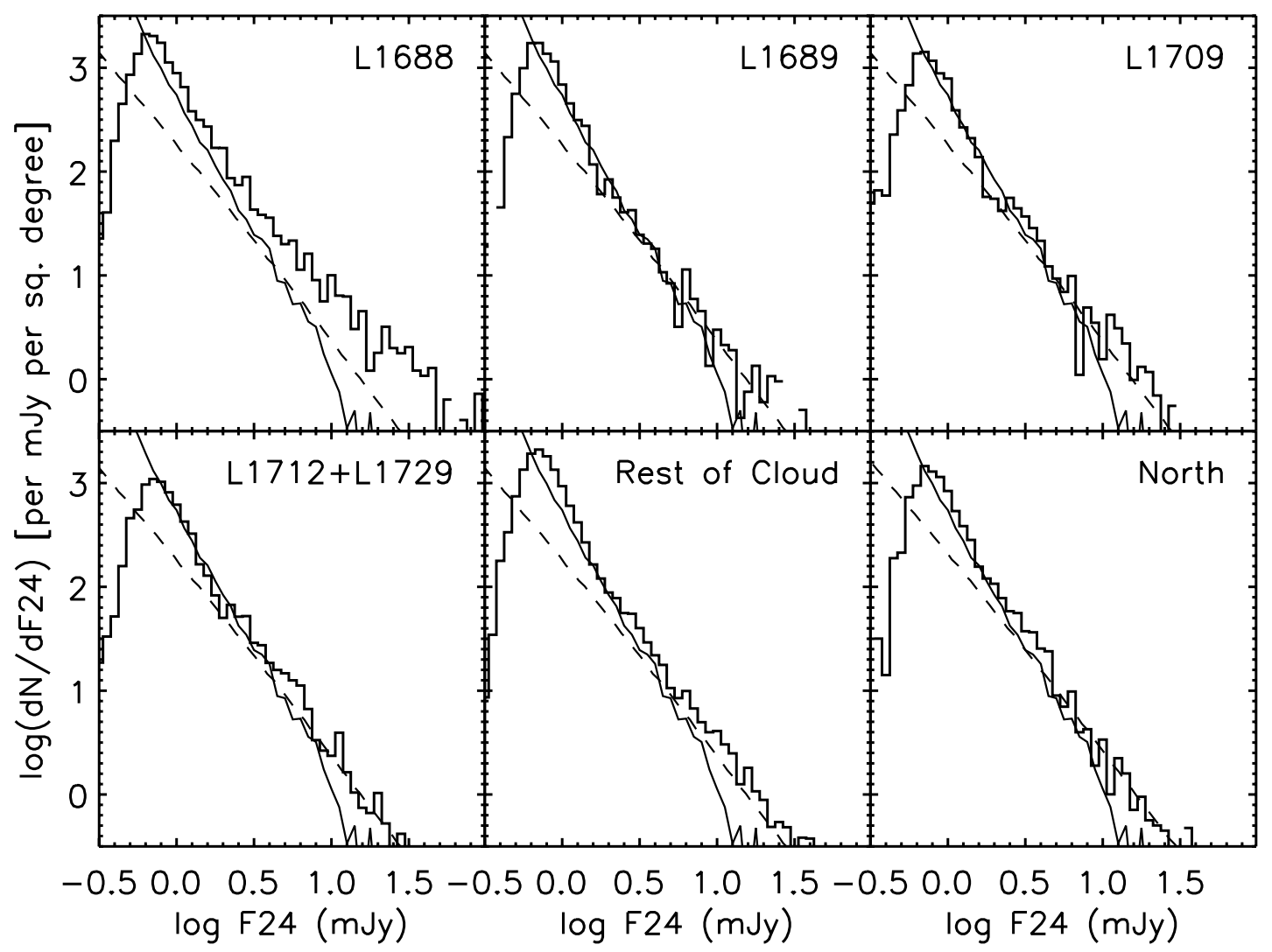

FIG. 8.-Ophiuchus differential number counts at $24 \mu \mathrm{m}$. The solid line shows the SWIRE ELAIS N1 extragalactic number counts, and the dashed line shows the Wainscoat model prediction of Galactic star counts.

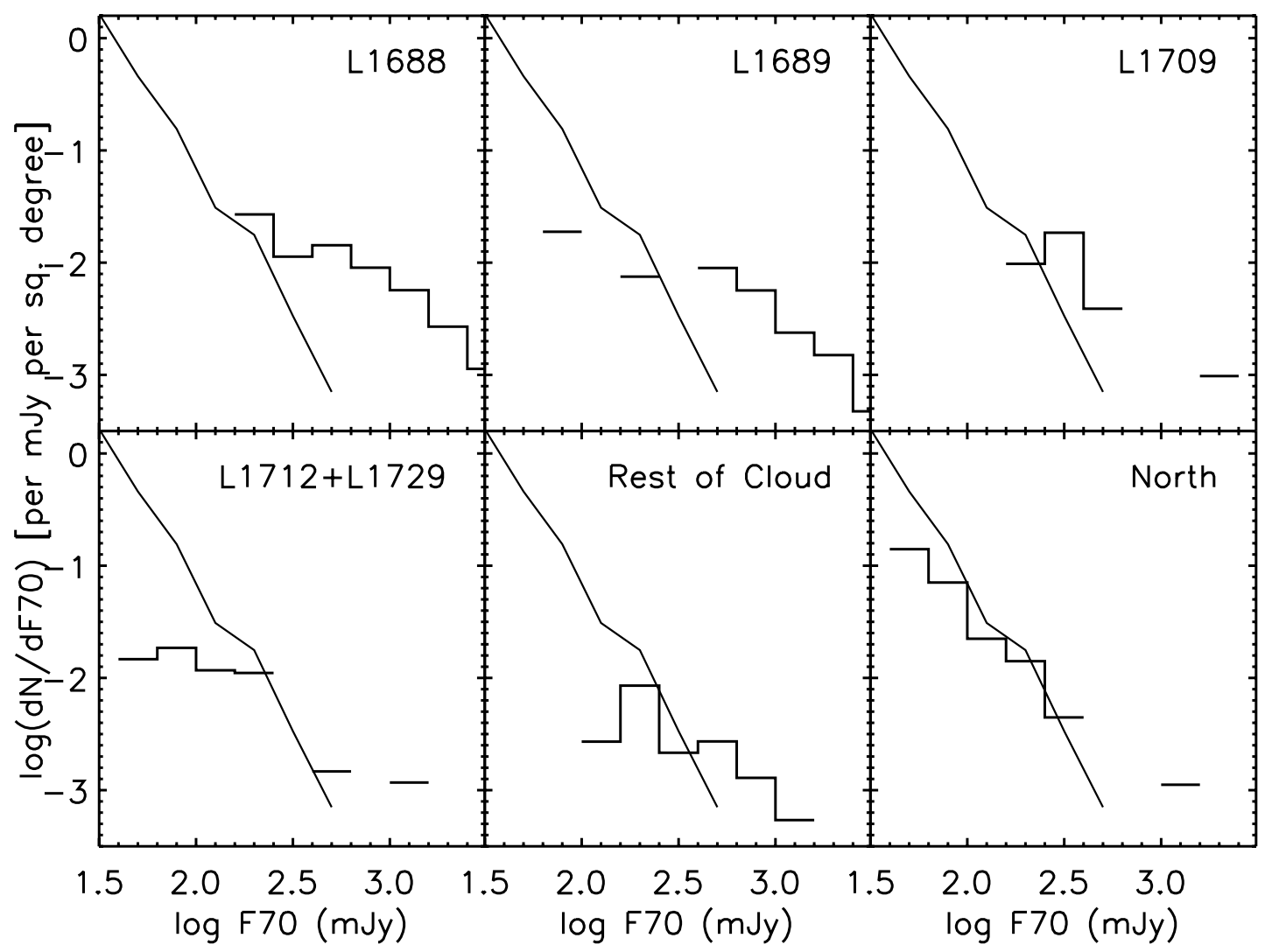

FIG. 9.- Ophiuchus differential number counts at $70 \mu \mathrm{m}$. The solid line shows the SWIRE ELAIS N1 extragalactic number counts. 
TABLE 3

Detected Sources

\begin{tabular}{|c|c|c|c|c|c|c|c|c|c|}
\hline Item & Overall & IRAC Coverage & L1688 & L1689 & L1709 & L1712+L1729 & Rest of Cloud & North & Off-Cloud \\
\hline No. $70 \mu \mathrm{m}$ objects & 81 & 31 & 24 & 12 & 5 & 12 & 14 & 13 & 2 \\
\hline No. 24 and $70 \mu \mathrm{m}$ objects................................... & 50 & 31 & 13 & 6 & 4 & 5 & 11 & 11 & 0 \\
\hline No. 24,70 , and $160 \mu \mathrm{m}$ objects ..................... & 5 & 4 & 1 & 1 & 1 & 1 & 0 & 1 & 0 \\
\hline 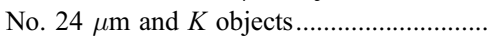 & 2590 & 1197 & 297 & 195 & 149 & 539 & 1102 & 308 & 177 \\
\hline No. $160 \mu \mathrm{m}$ and any IRAC objects............. & 6 & 6 & 1 & 1 & 2 & 1 & 0 & 1 & 0 \\
\hline
\end{tabular}

$2.6^{\prime} \times 0.97^{\prime}$. The Dish Surface Optimization System was used throughout the run, providing a stable beam, with a FWHM of $\sim 8^{\prime \prime}$ at $350 \mu \mathrm{m}$. Submillimeter conditions were excellent throughout the run, with $\tau_{225}$ ranging from 0.03 to 0.045 . Target observations were interspersed with observations of IRAS 16293-2422 and Callisto, which were used for both pointing and flux correction. The data were reduced using the CSO data pipeline CRUSH and relative aperture photometry on the sources obtained. The measurement uncertainties include the flux calibration uncertainty, which is typically on the order of $20 \%$.

\section{RESULTS}

\subsection{Multiwavelength Images}

The MIPS $24 \mu \mathrm{m}$ mosaic (Fig. 2) shows a great deal of diffuse nebulosity and many bright point sources. The central region of L1688 (Fig. 3) is dominated by nebulosity near the early-type stars (S1 and SR 3) embedded in the cloud. The S1 source is saturated in the MIPS $24 \mu \mathrm{m}$ image, but the peak appears extended, and it is surrounded by a complex cometary nebula opening toward the northeast. The B star SR 3 also appears as a resolved nebulosity at $24 \mu \mathrm{m}$, as do the more embedded early-type objects WL 16 and WL 22. The WL 22 nebula has a central core with a FWHM of $18^{\prime \prime}$ and an outer cometary morphology extending to radii of $30^{\prime \prime}$. The WL 16 nebula is more elongated, $2^{\prime} \times 35^{\prime \prime}$ along a position angle (P.A.) of $10^{\circ}-50^{\circ}$ from the major axis of the polycyclic aromatic hydrocarbon disk reported by Ressler \& Barsony (2003). An elliptical ring of emission about $1^{\prime}$ across is centered on a point source near the position of ISO-Oph 125 and ISOOph 124 (YLW 12B and 12A; spectral types F7 and M4; Greene \& Meyer 1995; Luhman \& Rieke 1999). Two other point sources within this ring are also detected at the positions of ISO-Oph 128 and ISO-Oph 129. South of these sources, the HD 147889 star appears only as a faint point source against some of the brightest parts of the central Oph nebulosity and shows a purely photospheric SED. This confirms that the large FIR excesses assigned to this source by the IRAS Point Source Catalog (PSC) are most likely due to interstellar emission, rather than circumstellar material. Ground-based photometry of this source (Wilking et al. 1989) also has suggested a lack of circumstellar material in this system. However, HD 148579 (B9 V), south of L1689, has a resolved $24 \mu \mathrm{m}$ nebula, as does the nearby HD 148605 (B3 V). Interestingly, these stars, which are apparently adjacent to or embedded in cloud material, have not excited the interstellar nebulosity to the same degree associated with the early-type stars in the central cluster. The $24 \mu \mathrm{m}$ mosaic also shows a surprising amount of "dark" nebulosity (i.e., absorbing perhaps $80 \%$ of the background illumination in the vicinity of the L1688 cluster and extending toward L1689). One of the $24 \mu \mathrm{m}$ dark cloudlets is shown in Figure 4.

The longer wavelength MIPS mosaics are shown in Figures 5 and 6. The $70 \mu \mathrm{m}$ mosaic still shows striations from the detector history effects which are not entirely removed in the pipeline processing for each scan leg. Contributing to this appearance is the fact that the map was filled by interleaving observations at two different epochs. Despite the cosmetic imperfections, the MIPS $70 \mu \mathrm{m}$ map clearly shows that the diffuse emission at this wavelength is brightest in the L1688 region and in the area extending from northwest to southwest of the central cluster. The COMPLETE CO maps of the Oph clouds (Ridge et al. 2006) show that this region of Oph West consists of generally tenuous cloud material with a few interspersed clumps. It is possible that these regions are bright in the long-wavelength MIPS bands because they are on a direct line of sight to the young B stars in the central cluster and are heated by them. S1, WL 16, WL 22, and YLW 12B continue to be nebulous at $70 \mu \mathrm{m}$ as they are at $24 \mu \mathrm{m}$. There is also a small area of diffuse nebulosity adjacent to HD 148605. The sources IRAS $16293-2422$ and IRAS $16288-2450$ are also prominent at this wavelength. The $160 \mu \mathrm{m}$ mosaic (Fig. 6) shows the same L1688 west dust emission features as the $70 \mu \mathrm{m}$ image. However, the cold dust in L1689 and the L1712+1729 filaments becomes prominent at this wavelength. Young et al. (2006) showed that there are some weak millimeter sources in these filaments. The central region of L1688 is saturated in the MIPS $160 \mu \mathrm{m}$ image. Figure 7 shows a three-color image with all three channels of MIPS included.

\subsection{Source Counts}

MIPS source counts in Ophiuchus are a combination of cloud members, foreground/background stars in the galaxy, and the extragalactic background. An excess of sources above comparison regions outside the clouds implies the presence of young stars with disks. Figure 8 shows the observed Ophiuchus $24 \mu \mathrm{m}$ differential source counts in comparison to observed source counts from the SWIRE ELAIS N1 extragalactic field (solid lines) and to the prediction for Galactic star counts in the IRAS $25 \mu \mathrm{m}$ band from the Wainscoat \& Cowie (1992) model provided by J. Carpenter (2001, private communication; dashed lines). Aside from L1688, all of the other regions have negligible levels of excess $24 \mu \mathrm{m}$ source counts, indicating that only small numbers of young stars are present in these fields. L1709 has a modest excess above $10 \mathrm{mJy}$. These plots indicate that only L1688 has a substantial young population relative to the various foreground/background sources expected in the field.

Figure 9 shows $70 \mu \mathrm{m}$ source counts in Ophiuchus, along with extragalactic background counts from the SWIRE ELAIS N1 


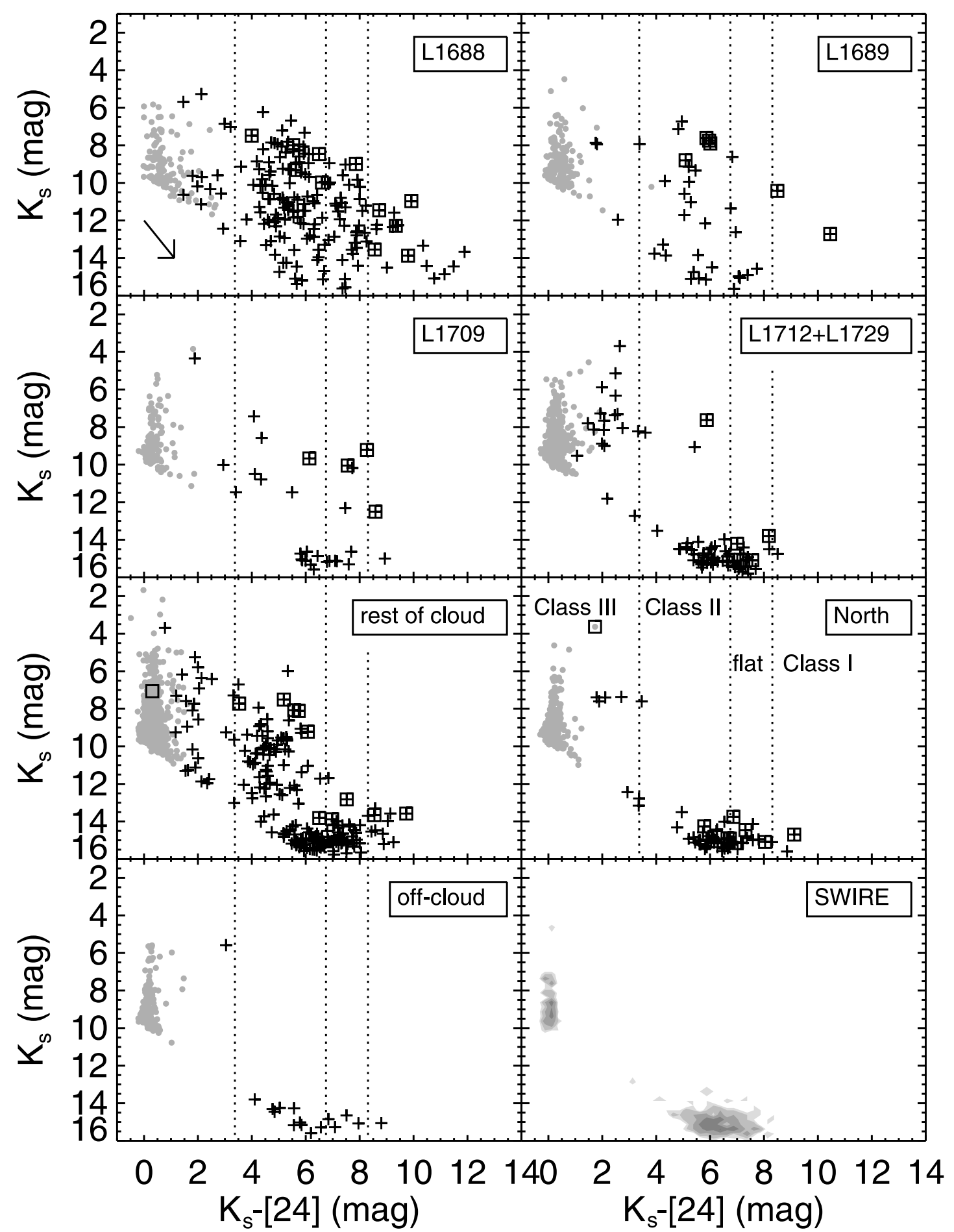

FIG. 10.- $K_{\mathrm{s}}$ vs. $K_{\mathrm{s}}-[24]$ for Ophiuchus objects in the various subclusters as marked, the "rest of the cloud," the off-cloud region, and SWIRE (contour plot, bottom right). Objects in SWIRE are expected to be mostly galaxies (objects with $K_{s} \gtrsim 14$ ) or plain photospheres (objects with $K_{s}-[24] \lesssim 1$; marked as gray on the Oph region plots). Boxed sources are also detected at $70 \mu \mathrm{m}$. The vertical dashed lines delineate (left to right) regions where Class I, flat-spectrum, Class II, and Class III infrared source models would reside.

field. At the low sensitivity of our $70 \mu \mathrm{m}$ measurements, detection of normal stellar photospheres will be negligibly rare; thus, comparisons with Galactic star counts are not needed. Clearly, given the high backgrounds and small number of cataloged $70 \mu \mathrm{m}$ sources, we are dealing with small-number statistics. However, the plots do show significantly more sources at bright flux density levels for the regions L1688, L1689, and possibly L1709 than would be found from the extragalactic background. For L1688, the excess sources extend down to $200 \mathrm{mJy}$, while for the other two regions, the excess above background starts at about $300 \mathrm{mJy}$. In the extended cloud outside the clusters, a slight excess in source counts might be present above a flux density of $400 \mathrm{mJy}$. Note that the depth of $70 \mu \mathrm{m}$ extractions is greatest in the Oph North field, where the diffuse background is lowest. In this field the $70 \mu \mathrm{m}$ source counts follow the SWIRE survey curve to the point where the greater SWIRE sensitivity diverges from the $\mathrm{c} 2 \mathrm{~d}$ sensitivity. 


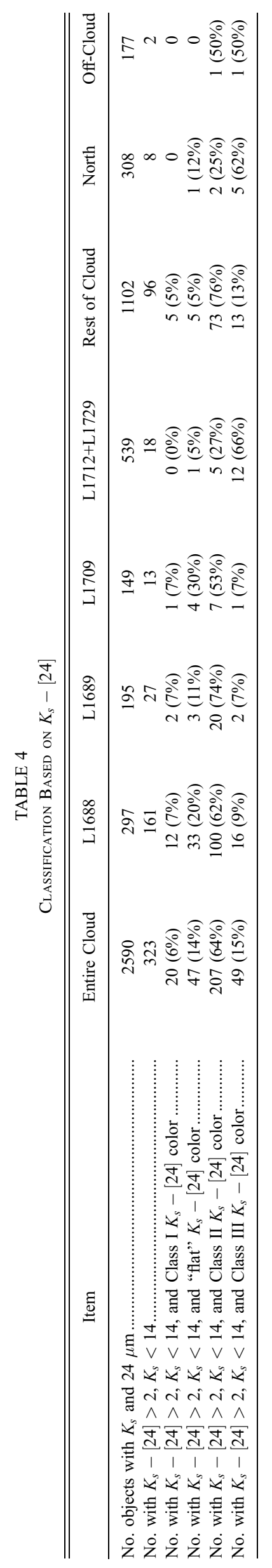




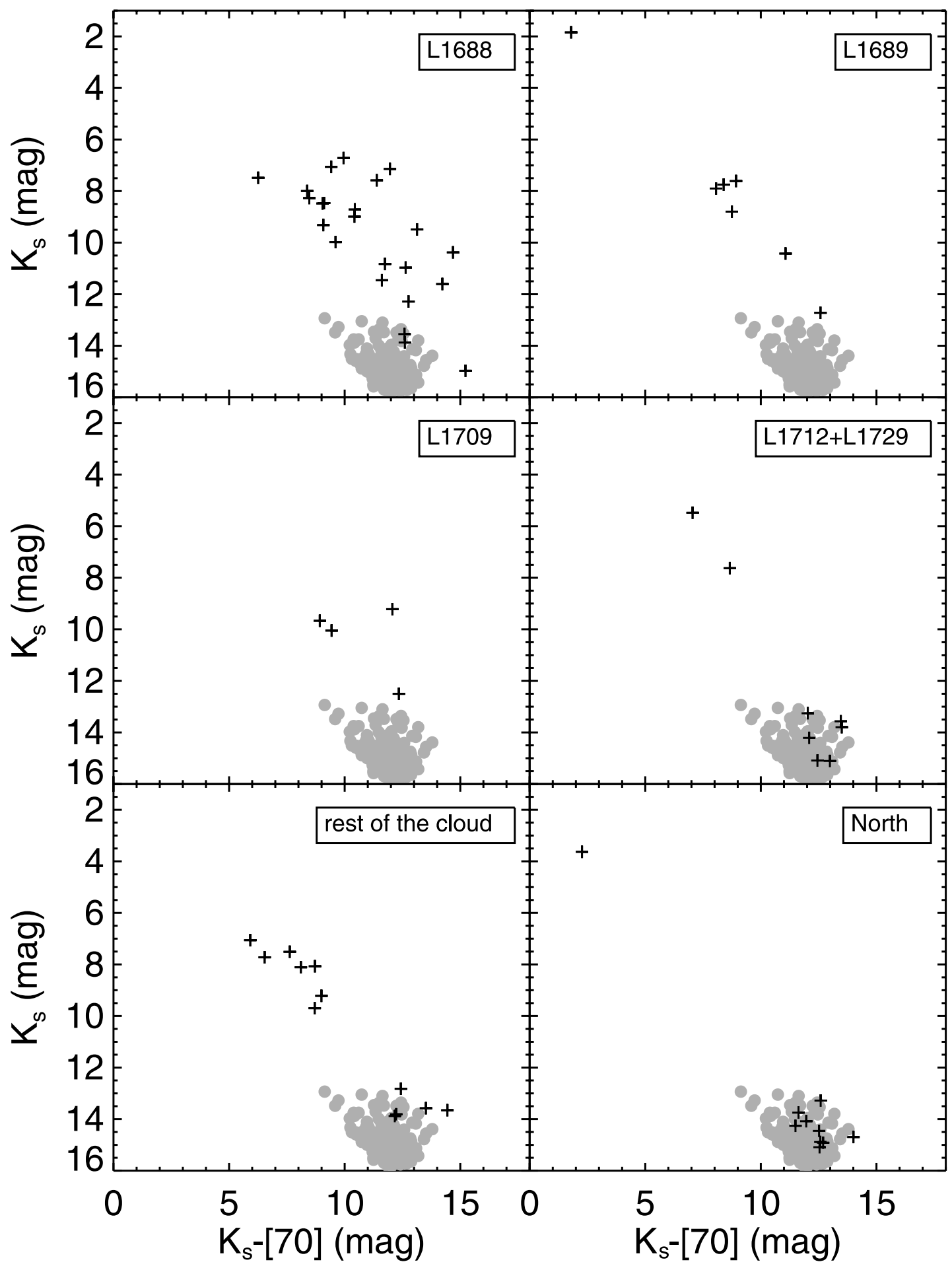

FIG. 11. $-K_{s}$ vs. $K_{s}-[70]$ color-magnitude diagram for various regions in Oph (crosses), with data for SWIRE ( gray circles) included for comparison. See text for details.

\subsection{Band-Merging with IRAC and 2MASS}

The IRAC data for the $\mathrm{c} 2 \mathrm{~d}$ Ophiuchus field (covering $\sim 6.8 \mathrm{deg}^{2}$ ) will be described in L. E. Allen et al. (2008, in preparation), and these data (as band-merged with the MIPS sources) were included in our final catalog. Our MIPS map covers more than twice the area of the IRAC map. In the $A_{V}>5$ region with full MIPS and IRAC coverage, there are 2608 MIPS- 24 point sources, $88 \%$ of which have a counterpart in at least one IRAC band; $3170 \mu \mathrm{m}$ point sources, all but one of which have an IRAC counterpart; and six
$160 \mu \mathrm{m}$ point sources, all with IRAC counterparts. The wide variation in resolution between IRAC $\left(\sim 2^{\prime \prime}\right.$ at $\left.3.6 \mu \mathrm{m}\right)$ and MIPS ( $6^{\prime \prime}$ at $24 \mu \mathrm{m}, 18^{\prime \prime}$ at $70 \mu \mathrm{m}$, and $36^{\prime \prime}$ at $160 \mu \mathrm{m}$ ) leads to potential confusion of several IRAC objects within a single MIPS beam. Details of the band-merger are given in the c2d delivery documents. ${ }^{16}$ For the total MIPS map region, the above source

\footnotetext{
${ }^{16}$ See the information from R. Cutri et al. at http://irsa.ipac.caltech.edu/ applications/Gator/.
} 


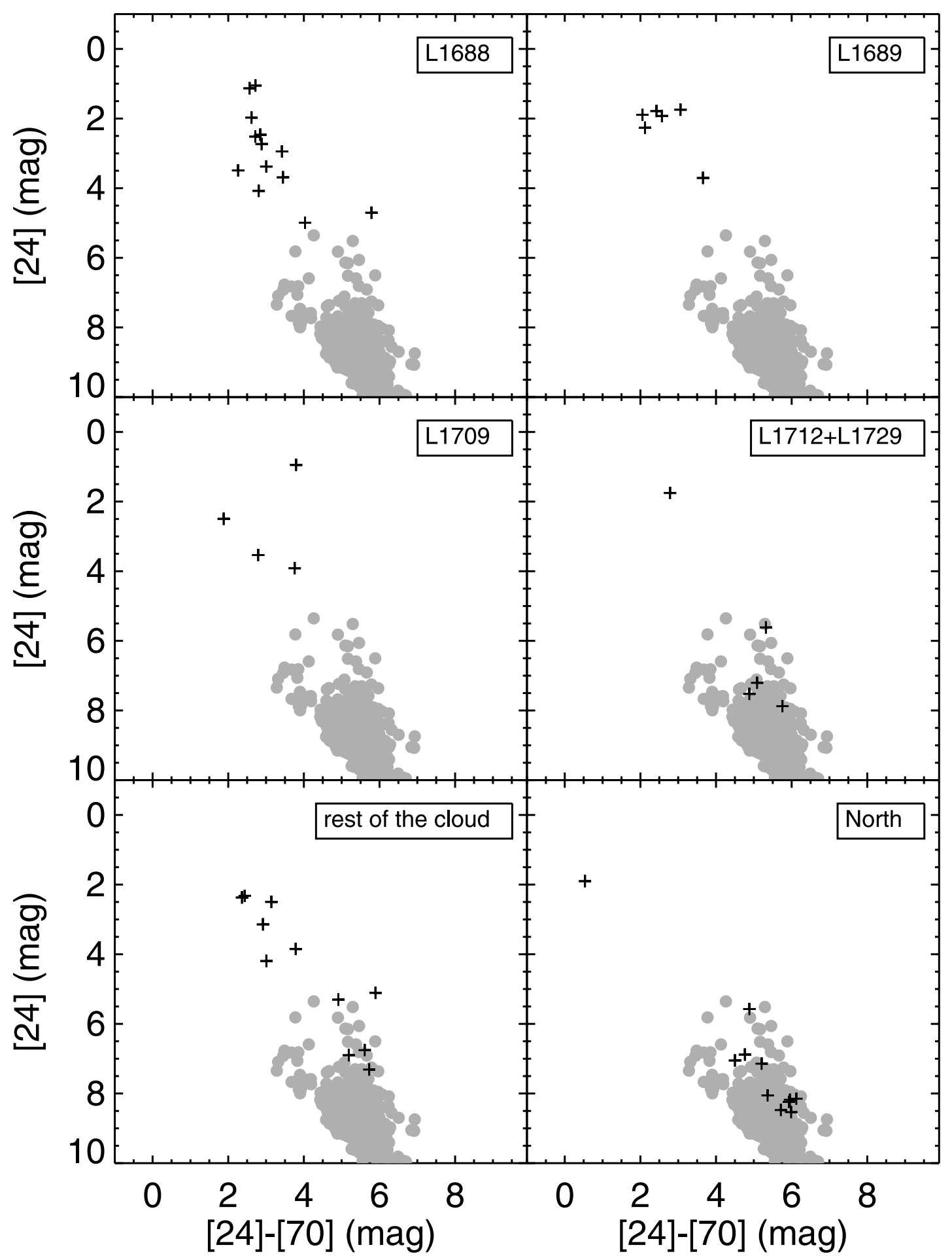

FIG. 12.-A [24] vs. [24] - [70] color-magnitude diagram for Ophiuchus (crosses), with data for SWIRE ( gray circles) included for comparison. As in Fig. 11, the two clumps correspond to extragalactic $([24] \sim 7-10)$ and YSO candidates $([24] \lessgtr 5)$, and there are few extragalactic objects in the clusters because the high nebulosity limits the depth of the survey. Very red sources $([24]-[70]>6)$ are the most embedded objects.

counts approximately double (see Table 3 ). The c2d source catalog containing all Oph Spitzer sources can be found online. ${ }^{17}$

Near-IR $J H K_{s}$ data from $2 \mathrm{MASS}^{18}$ were also used in constructing our catalog. The $2 \mathrm{MASS}$ data obviously covered the entire region of the MIPS map, but to a shallower depth than the IRAC observations, so only $45 \%$ of the MIPS- 24 sources have 2 MASS

17 See http://data.spitzer.caltech.edu/popular/c2d/20051220_enhanced_v1/Oph/.

${ }^{18}$ See the c2d delivery documents by N. J. Evans II et al. online.
$K$ counterparts, similar to the fraction seen in the Cham II catalog (Young et al. 2005).

\subsection{Color-Magnitude and Color-Color Plots}

For relatively bright sources, $K_{s}$ versus $K_{s}-[24]$ can be used to separate potential YSOs from stars and most extragalactic sources. This metric has been used in a variety of Spitzer papers to identify stars with infrared excess (Gorlova et al. 2004; Padgett et al. 2004, 2006; Rebull et al. 2007; etc.). Stellar photospheres 


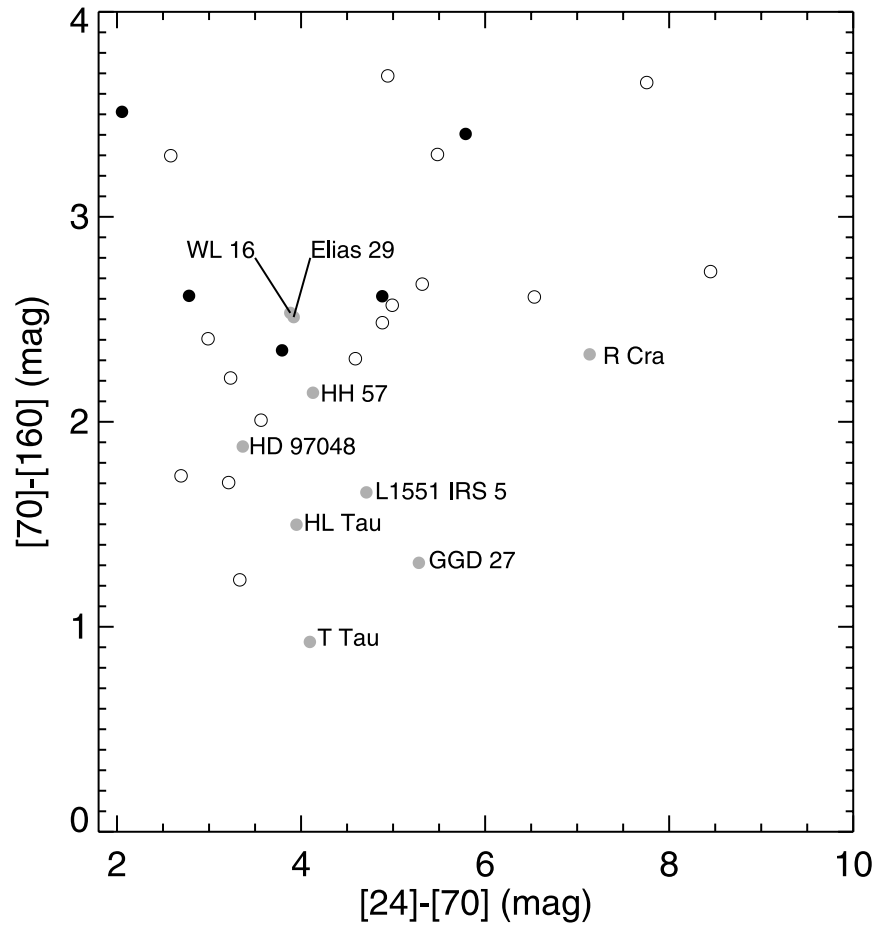

FIG. 13.-Color-color diagram for sources detected but not saturated in all three MIPS bands. Objects shown are from the current Ophiuchus survey (black circles), the c2d MIPS Perseus survey (open circles; Rebull et al. 2007), and bright objects studied by ISO (gray circles; A. Noriega-Crespo 2005, private communication).

without excess are clustered around $K_{s}-[24]=0$. If $K_{s}-$ [24] $>2$, then a strong excess is present at $24 \mu \mathrm{m}$. Most extragalactic sources are fainter than $K_{s}=14$.

Figure 10 presents $K_{s}$ versus $K_{s}-$ [24] color-magnitude diagrams for L1688, L1689, L1709, the L1712+L1729 filament, Oph North, the rest of the cloud, and the extragalactic SWIRE ELAIS N1 field. Note that sources that appear to be pure photospheres appear in gray. SWIRE $24 \mu \mathrm{m}$ sources tend to congregate in two groups, namely, main-sequence photospheres with colors of zero and galaxies with faint $K$ magnitudes and colors of $6-8$. Thus, $K_{s}-[24]$ plots help to define regions of colormagnitude space which are likely to be affected by extragalactic sources seen through the cloud. In Figure 10 dashed lines (right to left) denote the divisions between Class I, flat, Class II, and Class III objects following Rebull et al. (2007); in brief, we can use the observed $K_{s}-[24]$ colors and assign an $\alpha$-index following Greene et al. (1994). Note that the formal Greene et al. classification puts no lower limit on the colors of Class III objects (thereby including those with SEDs resembling bare stellar photospheres, and allowing for other criteria to define youth). In our case, since we know little about many of these objects, in an attempt to limit contamination from foreground/background stars (and background galaxies) we have imposed the additional constraints that $K_{s}-[24]>2$ and $K_{s}<14$. Note that there are certainly true young cluster members that do not meet these (conservative) criteria, but identifying them (and separating them from the galaxies) is beyond the scope of this paper. L. E. Allen et al. (2008, in preparation) will discuss the combined IRAC and MIPS SEDs and present a list of cloud members.

Interstellar and circumstellar reddening could plausibly affect the location of sources on the $K_{s}$ versus $K_{s}-$ [24] diagram. To test this possibility, we used the extinction law derived from Spitzer lines of sight through Ophiuchus (Flaherty et al. 2007) to produce
TABLE 5

IRAS Results in the MIPS Ophiuchus Map (Main+North)

\begin{tabular}{|c|c|c|}
\hline Item & At $24 \mu \mathrm{m}$ & At $70 \mu \mathrm{m}$ \\
\hline $12 \mu \mathrm{m}$ PSC real (IRAS qual=3) detections ................ & 100 & 91 \\
\hline Cleanly retrieved & $74(74 \%)$ & $25(27 \%)$ \\
\hline 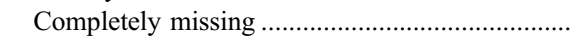 & $2(2 \%)$ & $41(45 \%)$ \\
\hline 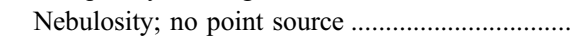 & $15(15 \%)$ & $24(26 \%)$ \\
\hline Resolved as multiple & $9(9 \%)$ & $1(1 \%)$ \\
\hline $25 \mu \mathrm{m}$ PSC real (IRAS qual=3) detections ................ & 63 & 63 \\
\hline Cleanly retrieved & $41(65 \%)$ & $26(41 \%)$ \\
\hline Completely missing & $1(1 \%)$ & $12(19 \%)$ \\
\hline 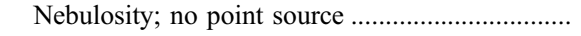 & $15(23 \%)$ & $24(38 \%)$ \\
\hline Resolved as multiple & $6(9 \%)$ & $1(1 \%)$ \\
\hline $60 \mu \mathrm{m}$ PSC real (IRAS qual=3) detections ................ & 93 & 84 \\
\hline Cleanly retrieved & $23(24 \%)$ & $20(23 \%)$ \\
\hline 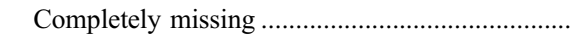 & $41(44 \%)$ & $16(19 \%)$ \\
\hline 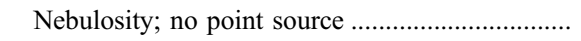 & $27(29 \%)$ & $48(57 \%)$ \\
\hline 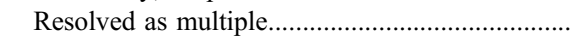 & $2(2 \%)$ & $0(0 \%)$ \\
\hline $100 \mu \mathrm{m}$ PSC real (IRAS qual=3) detections .............. & 55 & 50 \\
\hline 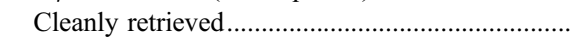 & $4(7 \%)$ & $3(6 \%)$ \\
\hline 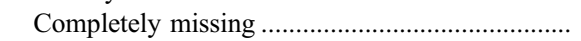 & $29(52 \%)$ & $12(24 \%)$ \\
\hline 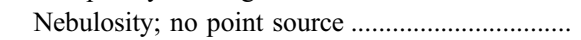 & $22(40 \%)$ & $35(70 \%)$ \\
\hline Resolved as multiple & $0(0 \%)$ & $0(0 \%)$ \\
\hline $12 \mu \mathrm{m}$ FSC real (IRAS qual=3) detections ................. & 50 & 35 \\
\hline 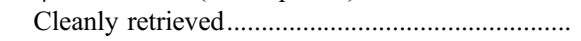 & $42(84 \%)$ & $3(8 \%)$ \\
\hline 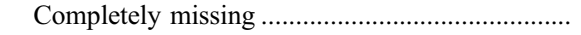 & $0(0 \%)$ & $24(68 \%)$ \\
\hline Nebulosity; no point source ……............................... & $7(14 \%)$ & $8(22 \%)$ \\
\hline Resolved as multiple & $1(2 \%)$ & $0(0 \%)$ \\
\hline $25 \mu \mathrm{m}$ FSC real (IRAS qual=3) detections ................. & 14 & 11 \\
\hline 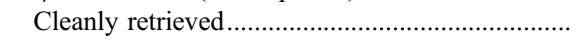 & $13(92 \%)$ & $5(45 \%)$ \\
\hline Completely missing ..... & $0(0 \%)$ & $6(54 \%)$ \\
\hline Nebulosity; $\mathrm{n}$ & $0(0 \%)$ & $0(0 \%)$ \\
\hline Resolved as multiple & $1(7 \%)$ & $0(0 \%)$ \\
\hline $60 \mu \mathrm{m}$ FSC real (IRAS qual=3) detections ................. & 0 & 0 \\
\hline $100 \mu \mathrm{m} \mathrm{FSC} \mathrm{real} \mathrm{(IRAS} \mathrm{qual=3)} \mathrm{detections} \mathrm{............}$ & 0 & 0 \\
\hline
\end{tabular}

a reddening vector in Figure 10. The vector depicts the effects of $5 \mathrm{mag}$ of $K$-band extinction, which moves a star toward the lower right. Note that unlike the SWIRE sources, which show a well-defined main sequence at $K_{s}-[24]=0$, almost all of the Ophiuchus sources show a displacement toward redder colors. Given the high extinctions throughout this region, many of the apparently small excess sources in L1712+L1729 and the "rest of cloud" areas may simply be reddened photospheres. In addition, Class II sources could plausibly be reddened into the Class I zone. Thus, in regions of very high extinction, classification of YSOs based on even long-wavelength MIPS data is complicated by reddening effects.

Table 4 provides statistics on the number of sources classified by $K_{s}-[24]$ as YSOs in the various subregions of the Ophiuchus map. The Oph North and off-cloud fields resemble the SWIRE regions, with most sources clumped in the photospheric and faint galaxy region of the diagram. The L1712+1729 filament shows a few more bright photospheres with small excesses than Oph North plus three potential Class II sources. L1709 and L1689 have a higher frequency of Class II sources than the filament, as well as a few flat and Class I SEDs. Although the number of sources in L1709 is small, this region actually has the highest fraction of Class I + flat spectrum sources in the region. These dense clouds also show fewer sources in the extragalactic clump at faint $K_{s}$ and large $K_{s}-[24]$ due to their small area and bright $24 \mu \mathrm{m}$ diffuse emission. Finally, the L1688 region has a high fraction of flat and Class I SED sources (27\%), and it dominates the region for sheer number of Class II sources (100). 
TABLE 6

IRAS PSC Detections not Recovered by Spitzer MIPS

\begin{tabular}{|c|c|c|}
\hline PSC Name & At $24 \mu \mathrm{m}$ & At $70 \mu \mathrm{m}$ \\
\hline $16169-2443$. & Missing (upper limit at 12,25 ) & Missing (detected at 60 , upper limit at 100 ) \\
\hline $16172-2503 \ldots \ldots \ldots \ldots \ldots \ldots \ldots . .$. & Missing (upper limit at 12,15 ) & Confused by nebulosity \\
\hline 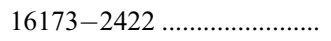 & Confused by nebulosity & Missing (detected at 60 , upper limit at 100 ) \\
\hline $16184-2452 \ldots \ldots \ldots \ldots \ldots \ldots \ldots . .$. & Confused by nebulosity & Confused by nebulosity \\
\hline 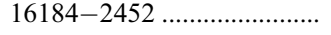 & Confused by nebulosity & Confused by nebulosity \\
\hline 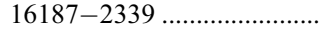 & Confused by nebulosity & Missing (upper limit at 60,100 ) \\
\hline 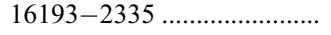 & Confused by nebulosity & Confused by nebulosity \\
\hline 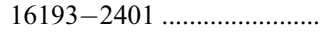 & Confused by nebulosity & Confused by nebulosity \\
\hline 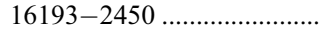 & Confused by nebulosity & Confused by nebulosity \\
\hline $16194-2410 \ldots \ldots \ldots \ldots \ldots \ldots \ldots . .$. & Missing (upper limit at 12,25 ) & Confused by nebulosity \\
\hline 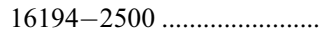 & Off edge & Confused by nebulosity \\
\hline 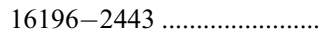 & Missing (upper limit at 12,25 ) & Confused by nebulosity \\
\hline $16200-2251 \ldots \ldots \ldots \ldots \ldots \ldots \ldots . .$. & Confused by nebulosity & Confused by nebulosity \\
\hline $16201-2410 \ldots \ldots \ldots \ldots \ldots \ldots \ldots . .$. & Retrieved (upper limit at 12) & Confused by nebulosity \\
\hline $16202-2356 \ldots \ldots \ldots \ldots \ldots \ldots \ldots \ldots$ & Missing (upper limit at 12,25 ) & Confused by nebulosity \\
\hline 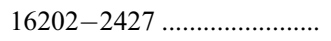 & Missing (upper limit at 12,25 ) & Missing (detected at 60 , upper limit at 100 ) \\
\hline $16203-2332 \ldots \ldots \ldots \ldots \ldots \ldots \ldots \ldots$ & Missing (upper limit at 12,25 ) & Missing (detected at 60, upper limit 100 ) \\
\hline $16205-2308 \ldots \ldots \ldots \ldots \ldots \ldots \ldots \ldots$ & Missing (upper limit at 12,25 ) & Confused by nebulosity \\
\hline $16209-2422 \ldots \ldots \ldots \ldots \ldots \ldots \ldots \ldots . .$. & Confused by nebulosity & Confused by nebulosity \\
\hline $16212-2316 \ldots \ldots \ldots \ldots \ldots \ldots . .$. & Missing (upper limit at 12,25 ) & Missing (detected at 60 , upper limit 100 ) \\
\hline $16214-2302 \ldots \ldots \ldots \ldots \ldots \ldots . .$. & Missing (upper limit at 12,25 ) & Confused by nebulosity \\
\hline $16214-2345 \ldots \ldots \ldots \ldots \ldots \ldots \ldots . .$. & Confused by nebulosity & Confused by nebulosity \\
\hline $16214-2436 \ldots \ldots \ldots \ldots \ldots \ldots \ldots . . .$. & Confused by nebulosity & Confused by nebulosity \\
\hline 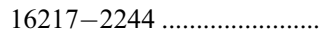 & Missing (upper limit at 12,25 ) & Confused by nebulosity \\
\hline $16219-2344 \ldots \ldots \ldots \ldots \ldots \ldots \ldots . .$. & Confused by nebulosity & Confused by nebulosity \\
\hline $16219-2417 \ldots \ldots \ldots \ldots \ldots \ldots \ldots . .$. & Confused by nebulosity & Confused by nebulosity \\
\hline $16221-2428 \ldots \ldots \ldots \ldots \ldots \ldots \ldots . .$. & Confused by nebulosity & Confused by nebulosity \\
\hline 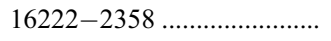 & Confused by nebulosity & Confused by nebulosity \\
\hline 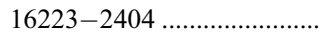 & Confused by nebulosity & Confused by nebulosity \\
\hline 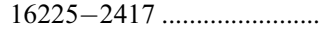 & Confused by nebulosity & Confused by nebulosity \\
\hline $16226-2319 \ldots \ldots \ldots \ldots \ldots \ldots \ldots$ & Broken into pieces & Confused by nebulosity \\
\hline $16226-2420 \ldots \ldots \ldots \ldots \ldots \ldots \ldots$ & Confused by nebulosity & Confused by nebulosity \\
\hline $16227-2418 \ldots \ldots \ldots \ldots \ldots \ldots \ldots . .$. & Confused by nebulosity & Confused by nebulosity \\
\hline $16228-2432 \ldots \ldots \ldots \ldots \ldots \ldots \ldots . . .$. & Confused by nebulosity & Confused by nebulosity \\
\hline $16229-2413 \ldots \ldots \ldots \ldots \ldots \ldots \ldots . .$. & Confused by nebulosity & Confused by nebulosity \\
\hline $16233-2421 \ldots \ldots \ldots \ldots \ldots \ldots \ldots . . .$. & Confused by nebulosity & Confused by nebulosity \\
\hline $16234-2436 \ldots \ldots \ldots \ldots \ldots \ldots \ldots . .$. & Confused by nebulosity & Confused by nebulosity \\
\hline $16234-2436 \ldots \ldots \ldots \ldots \ldots \ldots \ldots . .$. & Confused by nebulosity & Confused by nebulosity \\
\hline $16235-2416 \ldots \ldots \ldots \ldots \ldots \ldots \ldots \ldots$ & Retrieved & Confused by nebulosity \\
\hline $16238-2317 \ldots \ldots \ldots \ldots \ldots \ldots \ldots \ldots$ & Missing (upper limit at 12,25 ) & Confused by nebulosity \\
\hline 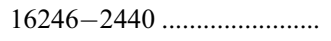 & Retrieved & Confused by nebulosity \\
\hline $16257-2533 \ldots \ldots \ldots \ldots \ldots \ldots \ldots \ldots$ & Missing (upper limit at 12,25 ) & Confused by nebulosity \\
\hline $16262-2545 \ldots \ldots \ldots \ldots \ldots \ldots \ldots . .$. & Retrieved & Missing (detected at 60 , upper limit at 100 ) \\
\hline $16265-2350 \ldots \ldots \ldots \ldots \ldots \ldots \ldots . .$. & Missing (upper limit at 12,25 ) & Confused by nebulosity \\
\hline 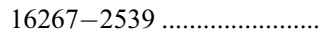 & Confused by nebulosity & Confused by nebulosity \\
\hline $16268-2533 \ldots \ldots \ldots \ldots \ldots \ldots \ldots . . .$. & Missing (upper limit at 12,25 ) & Missing (detected 60, upper limit at 100 ) \\
\hline $16269-2454 \ldots \ldots \ldots \ldots \ldots \ldots \ldots . .$. & Confused by nebulosity & Confused by nebulosity \\
\hline 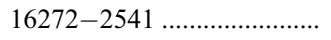 & Confused by nebulosity & Confused by nebulosity \\
\hline 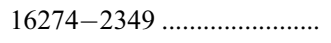 & Confused by nebulosity & Confused by nebulosity \\
\hline $16275-2251 \ldots \ldots \ldots \ldots \ldots \ldots \ldots .$. & Missing (upper limit at 12,25 ) & Missing (detected at 60 , upper limit at 100 ) \\
\hline $16277-2356 \ldots \ldots \ldots \ldots \ldots \ldots \ldots$ & Confused by nebulosity & Missing (detected at 60 , upper limit at 100 ) \\
\hline $16280-2353 \ldots \ldots \ldots \ldots \ldots \ldots \ldots . . .$. & Confused by nebulosity & Missing (detected at 60 , upper limit at 100 ) \\
\hline 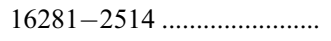 & Missing (upper limit at 12,25 ) & Confused by nebulosity \\
\hline $16285-2519 \ldots \ldots \ldots \ldots \ldots \ldots \ldots . .$. & Missing (upper limit at 12,25 ) & Confused by nebulosity \\
\hline $16289-2527 \ldots \ldots \ldots \ldots \ldots \ldots \ldots . .$. & Missing (upper limit at 12,25 ) & Confused by nebulosity \\
\hline $16301-2525 \ldots \ldots \ldots \ldots \ldots \ldots \ldots . .$. & Missing (upper limit at 12,25 ) & Confused by nebulosity \\
\hline $16303-2428 \ldots \ldots \ldots \ldots \ldots \ldots . . . .$. & Confused by nebulosity & Confused by nebulosity \\
\hline 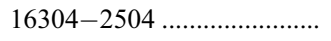 & Missing (upper limit at 12,25 ) & Confused by nebulosity \\
\hline $16306-2435 \ldots \ldots \ldots \ldots \ldots \ldots \ldots . .$. & Confused by nebulosity & Confused by nebulosity \\
\hline $16311-2419 \ldots \ldots \ldots \ldots \ldots \ldots \ldots . .$. & Confused by nebulosity & Confused by nebulosity \\
\hline $16313-2439 \ldots \ldots \ldots \ldots \ldots \ldots \ldots \ldots$ & Confused by nebulosity & Confused by nebulosity \\
\hline $16318-2519 \ldots \ldots \ldots \ldots \ldots \ldots \ldots . .$. & Missing (upper limit at 12,25 ) & Missing (detected at 60 , upper limit at 100 ) \\
\hline $16322-2421 \ldots \ldots \ldots \ldots \ldots \ldots \ldots . .$. & Confused by nebulosity & Confused by nebulosity \\
\hline $16325-2433 \ldots \ldots \ldots \ldots \ldots \ldots \ldots . .$. & Confused by nebulosity & Confused by nebulosity \\
\hline $16330-2424 \ldots \ldots \ldots \ldots \ldots \ldots \ldots . .$. & Missing (upper limit at 12,25 ) & Confused by nebulosity \\
\hline $16330-2431 \ldots \ldots \ldots \ldots \ldots \ldots \ldots$ & Missing (upper limit at 12,25 ) & Confused by nebulosity \\
\hline
\end{tabular}




\begin{tabular}{|c|c|c|}
\hline PSC Name & At $24 \mu \mathrm{m}$ & At $70 \mu \mathrm{m}$ \\
\hline 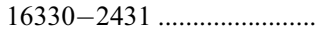 & Missing (upper limit at 12,25 ) & Confused by nebulosity \\
\hline $16335-2402 \ldots \ldots \ldots \ldots \ldots \ldots . . .$. & Missing (upper limit at 12,25 ) & Missing (detected at 60 , upper limit at 100 ) \\
\hline $16335-2419 \ldots \ldots \ldots \ldots \ldots \ldots . . .$. & Missing (upper limit at 12,25 ) & Confused by nebulosity \\
\hline 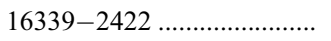 & Missing (upper limit at 12,25 ) & Confused by nebulosity \\
\hline 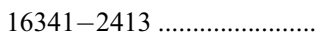 & Missing (upper limit at 12,25 ) & Missing (detected at 60 , upper limit at 100 ) \\
\hline $16342-2413 \ldots \ldots \ldots \ldots \ldots \ldots \ldots . . .$. & Missing (upper limit at 12,25 ) & Missing (detected at 60 , upper limit at 100 ) \\
\hline 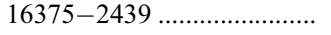 & Missing (upper limit at 12,25 ) & Confused by nebulosity \\
\hline 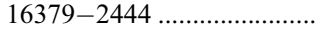 & Missing (upper limit at 12,25 ) & Missing (detected at 60 , upper limit at 100 ) \\
\hline $16383-2407$....................... & Missing (upper limit at 12 , detected at 25 ) & Missing (upper limit at 60,100 ) \\
\hline $16414-2337 \ldots \ldots \ldots \ldots \ldots \ldots \ldots . .$. & Missing (upper limit at 12,25 ) & Missing (detected at 60 , upper limit at 100 ) \\
\hline $16417-2155 \ldots \ldots \ldots \ldots \ldots \ldots \ldots . .$. & Confused by nebulosity & Confused by nebulosity \\
\hline 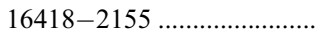 & Confused by nebulosity & Confused by nebulosity \\
\hline $16421-2146 \ldots \ldots \ldots \ldots \ldots \ldots . . .$. & Confused by nebulosity & Confused by nebulosity \\
\hline $16426-2129 \ldots \ldots \ldots \ldots \ldots \ldots . .$. & Confused by nebulosity & Confused by nebulosity \\
\hline 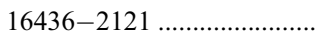 & Confused by nebulosity & Confused by nebulosity \\
\hline
\end{tabular}

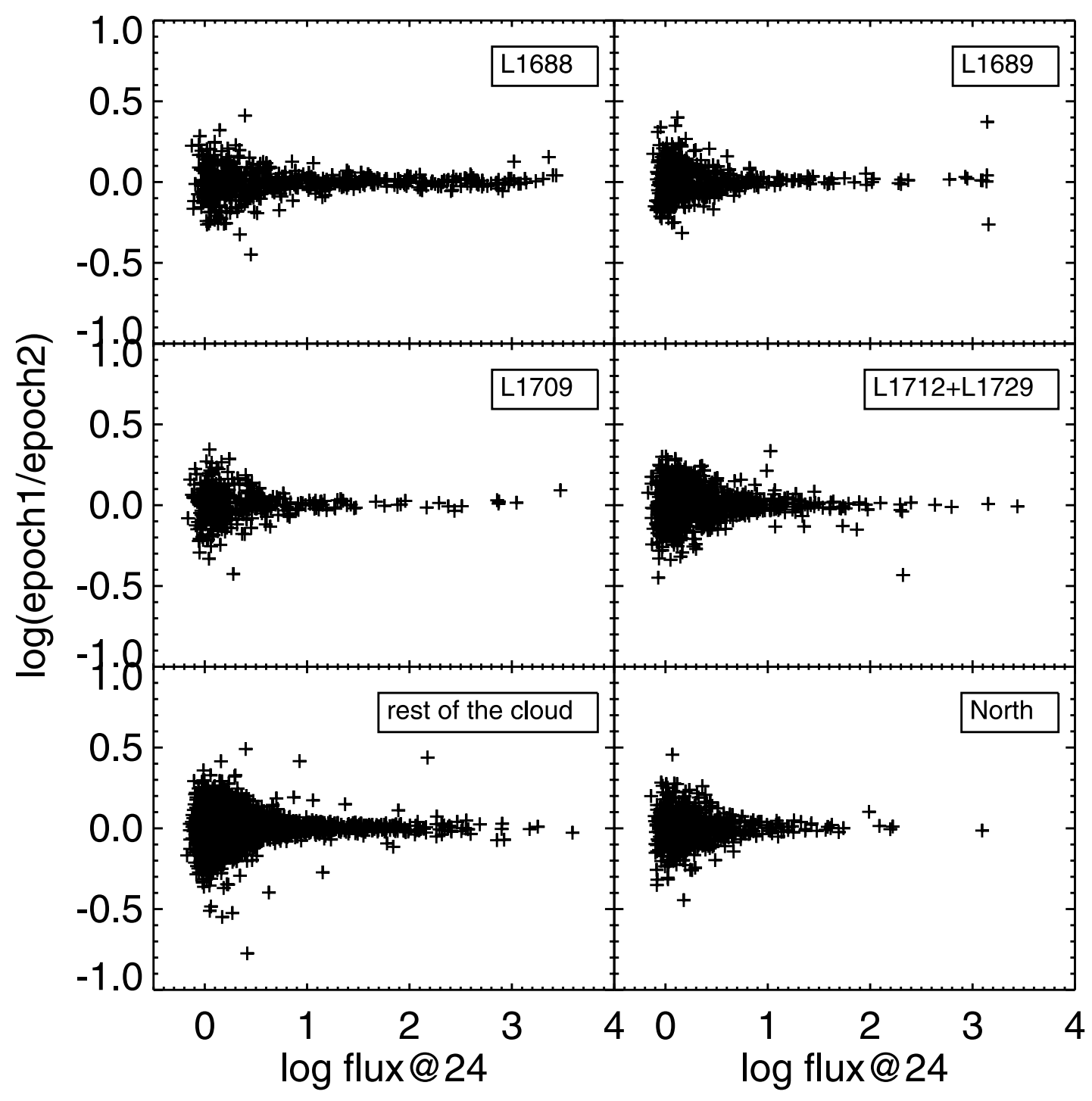

FIG. 14.- Variability of Ophiuchus $24 \mu \mathrm{m}$ sources between two observations separated by $3-8 \mathrm{hr}$. All cases of potential variability are well explained by instrumental effects. 


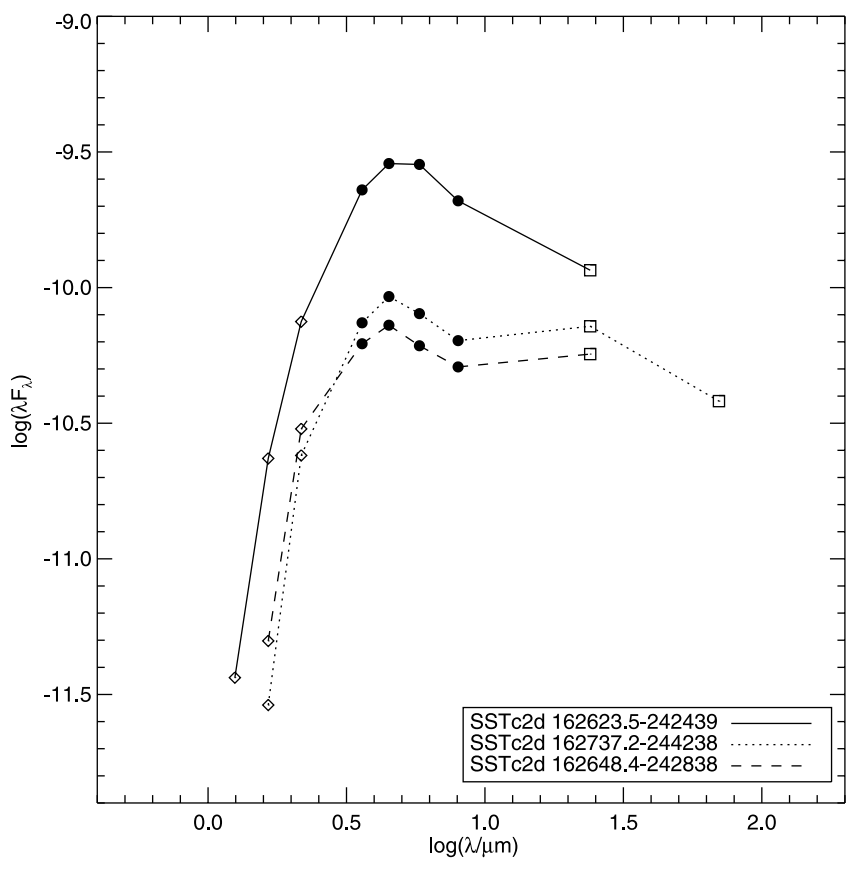

FIG. 15.- Infrared SEDs of several MIPS sources with $K_{s}-[24]>8$.

Figure 11 shows the $K_{s}$ versus $K_{s}-$ [70] plots for Ophiuchus. In this plot $K_{s}-[70]=0$ indicates a bare stellar photosphere. However, few if any bare stellar photospheres in this field are bright enough for detection in our very shallow MIPS $70 \mu \mathrm{m}$ survey. Extragalactic SWIRE sources from the ELAIS N1 field are shown for comparison ( gray circles). This plot is most useful for extremely bright YSOs which are saturated at $24 \mu \mathrm{m}$ but not $70 \mu \mathrm{m}$. Again, L1688 dominates the number of potential YSOs and has the most objects with large excesses. These are likely to be the youngest in the sample. Other regions have only a handful of $70 \mu \mathrm{m}$ YSOs, and the bulk are in the Class II regime with the exception of a few sources in L1709 and L1689. There is one object in L1689 with stellar or near-stellar colors out to $70 \mu \mathrm{m}$; it is SSTc2d 163528.6-245648, which we tentatively associate with Elias 2-83. This source may plausibly be a reddened star given the dark cloud visible in this area in the MIPS $24 \mu \mathrm{m}$ image. All the $70 \mu \mathrm{m}$ sources in Oph North are consistent with extragalactic background sources, except for SSTc2d 164417.8-220648, which can be identified with IRAS 16413-2201, a carbon star also known as NC 80 (Guglielmo et al. 1993).

Figure 12 presents a plot of [24] versus [24] - [70] for the Ophiuchus clouds. In the unlikely case of a bare stellar photosphere, $[24]-[70]=0$. Again, SWIRE sources are depicted as gray circles. Sources with large infrared excesses have large [24] - [70] colors. This color-magnitude diagram shows the separation of YSOs from extragalactic sources and emphasizes that extragalactic $70 \mu \mathrm{m}$ sources are only seen in the lower background regions. Although the number of sources seen in these plots is considerably smaller than in the $24 \mu \mathrm{m}$-only plots (due to the lower sensitivity of the MIPS $70 \mu \mathrm{m}$ channel), this colormagnitude diagram suggests confirmation of the picture of the L1688 cluster having a lower fraction of very young sources than L1709. However, it is true that a fair number of the most luminous MIPS sources in L1688 are saturated at $24 \mu \mathrm{m}(\geq \mathrm{Jy})$, thus eliminating them from these plots. In addition, the small number of sources in L1709 makes the comparison of questionable significance. Interestingly, some low-luminosity sources with large fractional excesses appear to be found outside of the described

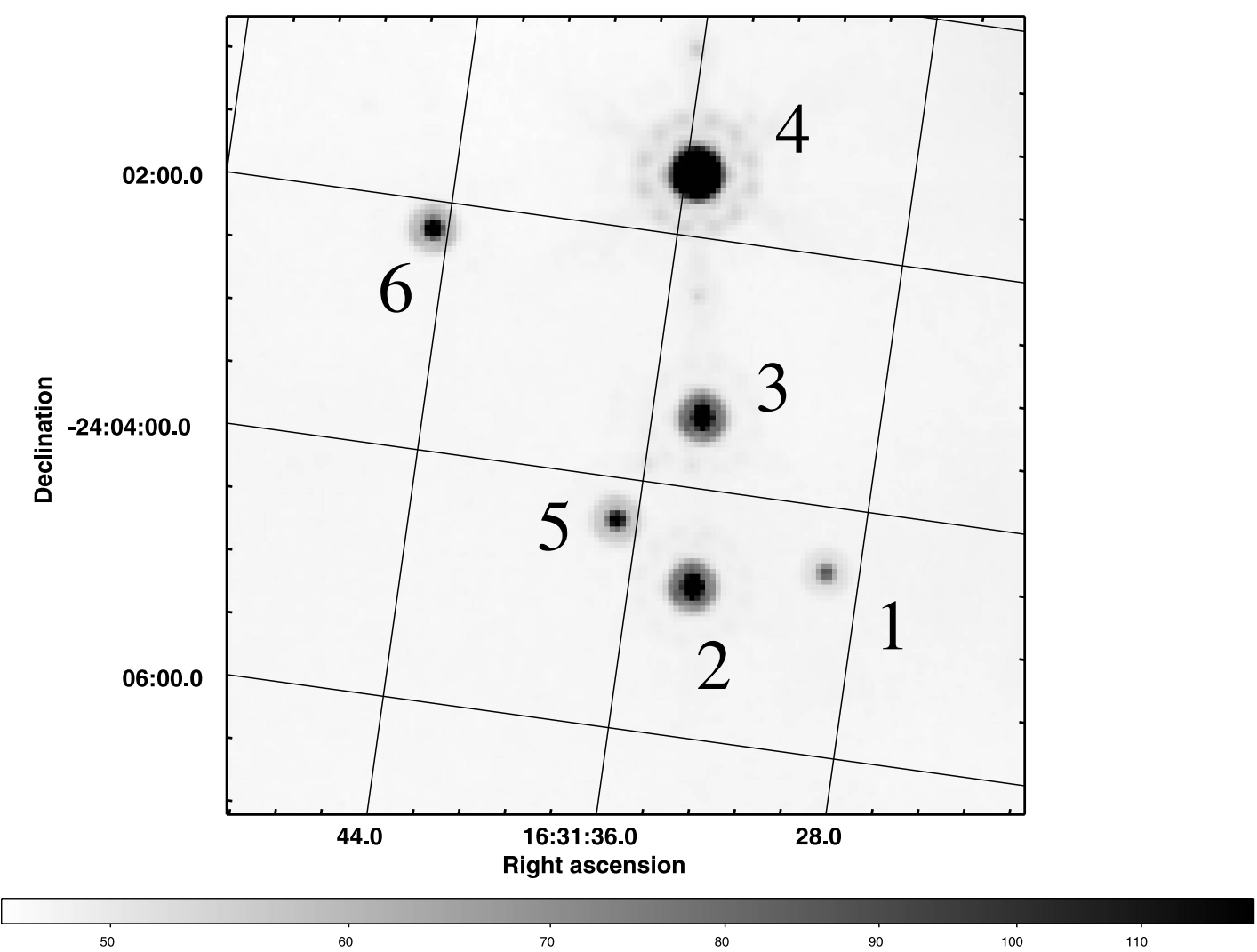

FIG. 16.-A $24 \mu \mathrm{m}$ image and finder chart for the L1709 aggregate. 
subclusters (in the "rest of the cloud" areas). Note that the brightest objects in these plots (those with [24] $\leq 5$ and [24] $-[70] \leq 6$ ) appear for reference in Table 7 below.

Unfortunately, due to very high backgrounds and saturation issues, as well as the difference in sensitivity between the various MIPS arrays, very few point sources (five) have measurable detections at all three MIPS bands. Figure 13 presents a plot of $[70]-[160]$ versus $[24]-[70]$. Included for comparison in this plot are colors calculated for MIPS bands from ISO SWS data for a variety of very famous well-studied embedded objects from A. Noriega-Crespo (2005, private communication); to facilitate comparison, these are the same objects used in a similar plot in Rebull et al. (2007). The four Ophiuchus sources all lie between [70] [160] of 2.5 and 3.5, while spanning a range of $4 \mathrm{mag}$ in [24] [70]. This may be an extinction effect, since three of the five objects are located in regions of Oph with apparently high extinction even at $24 \mu \mathrm{m}$.

\subsection{Comparison to IRAS}

As in Rebull et al. (2007) for Perseus, the MIPS data for Oph offer an opportunity to assess how successful the IRAS survey was in identifying point sources in a complex region. We now explicitly compare the IRAS PSC (Beichman et al. 1988) and IRAS Faint Source Catalog (FSC; Moshir et al. 1992) results in this region to the Spitzer c2d images and catalogs described in this paper. As for Perseus, extended emission is present in all three MIPS bands, posing a significant source of confusion for the large-aperture IRAS measurements. Many of the IRAS PSC objects were detected at 60 or $100 \mu \mathrm{m}$ only, with only upper limits at 12 and $25 \mu \mathrm{m}$; so even without Spitzer data, one might suspect that such sources might correspond to texture in the extended emission. As for Perseus (Rebull et al. 2007), the Spitzer data comparison clearly shows that significantly fewer spurious sources appear in the FSC than in the PSC.

A summary of the comparison of MIPS sources to IRAS sources is found in Table 5. For each IRAS PSC and FSC band, the number of IRAS detections are listed, followed by the number of these IRAS detections recovered by the MIPS 24 and $70 \mu \mathrm{m}$ arrays, IRAS detections not found by MIPS, IRAS detections determined to be nebulosity by MIPS, and IRAS detections resolved as multiple by MIPS. It is not surprising that few of the longwavelength IRAS sources are recovered by MIPS $24 \mu \mathrm{m}$. About $65 \%$ of the $25 \mu \mathrm{m}$ PSC sources are recovered as point sources in MIPS-24; only $23 \%$ of the $60 \mu \mathrm{m}$ PSC sources are recovered at MIPS-70. The most frequent reason for sources not being retrieved is confusion by nebulosity; $23 \%$ of the $25 \mu \mathrm{m}$ PSC sources and $57 \%$ of the $60 \mu \mathrm{m}$ PSC sources are so affected. Surprisingly few objects are resolved into multiple sources by Spitzer. Only one $25 \mu \mathrm{m}$ PSC detection is completely missing from MIPS-24; 16 of the $60 \mu \mathrm{m}$ PSC detections are completely missing from MIPS-70. A list of IRAS PSC sources not cleanly recovered appears in Table 6.

The FSC does a much better job of finding short-wavelength point sources, and is much less confused by the nebulosity. However, the FSC does not contain any sources on the west side of the cloud due to high surface brightness in the IRAS images. All but one of the $25 \mu \mathrm{m}$ FSC sources are recovered by Spitzer at $24 \mu \mathrm{m}$; the remaining source is resolved as multiple sources when viewed by MIPS. There are no $60 \mu \mathrm{m} \mathrm{FSC} \mathrm{high-quality} \mathrm{point} \mathrm{sources} \mathrm{in}$ this region.

While it would be interesting to study long-term far-infrared source variability in Oph, this is very difficult in practice. The vastly larger IRAS beam means that point-source flux densities can include large contributions from bright background nebu-

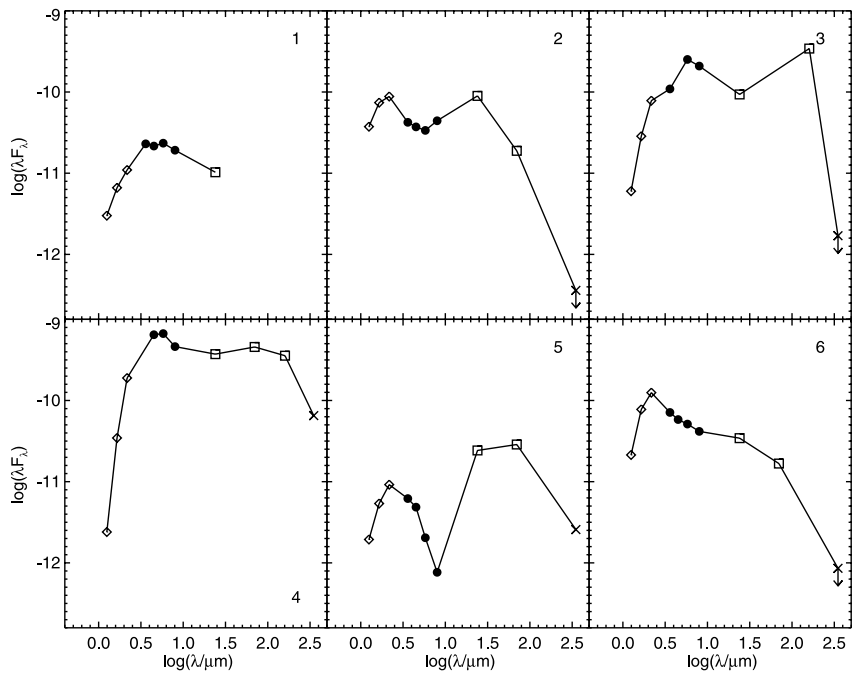

FIG. 17.- SEDs for six stars bright at $24 \mu \mathrm{m}$ found in the newly identified L1709 aggregate.

losity. The different calibration approaches of the two instruments require that any flux density comparison be made only after large color corrections are applied - corrections that can only be made accurately if the spectral slope is known across the photometric bandpasses. In addition, many sources detected by IRAS at $25 \mu \mathrm{m}$ are near the upper end of the MIPS $24 \mu \mathrm{m}$ dynamic range, where instrument calibration is less secure. For these reasons, we make no attempt to compare the MIPS and IRAS photometry for the sources detected in both surveys.

\subsection{Time Variability}

Because we have two epochs of observation at $24 \mu \mathrm{m}$, we can look for time variability on timescales of a few hours. Figure 14 shows the ratio of flux densities determined in the two epochs as a function of mean $24 \mu \mathrm{m}$ flux densities. It can be seen that typical variability is $\sim 10 \%$, consistent with our reported measurement uncertainty. There are four points whose flux ratio (logarithmic) is greater than 0.45 , and two whose flux ratio is less than -0.5 . Out of these six sources, three fall on the edge of the map, and the apparent variability is clearly due entirely to the low redundancy and signal-to-noise ratio at the ends of scan legs. Three additional objects fall in the L1688 region, where bright sources cause the readout-dependent gain variation known as "jailbars." Two of the L1688 objects are faint and located right on top of some strong jailbars, and one is deep within the bright, extended emission. Therefore, the apparent variability in all cases is entirely due to instrumental effects.

\section{DISCUSSION}

\subsection{Individual Objects}

\subsubsection{Young Stellar Objects with Extreme $K_{s}-[24]$ Colors}

YSO modeling indicates that some of the earliest evolutionary states of star/disk systems will be detected by Spitzer as point sources with extremely red colors (Robitaille et al. 2006). A search through our catalog for sources with $K_{s}<12$ and $K_{s}-$ [24] $>8$ yielded eight sources. Their SEDs are presented in Figure 15. One of these objects (SSTc2d 162623.5-242439) had been previously identified as CRBR 2322.1-1754 (Comeron et al. 1993). In that paper the source was detected out to the $Q$ band, but was not characterized further. In our data this source is probably confused at $24 \mu \mathrm{m}$ with GY 21, a flat-spectrum YSO 


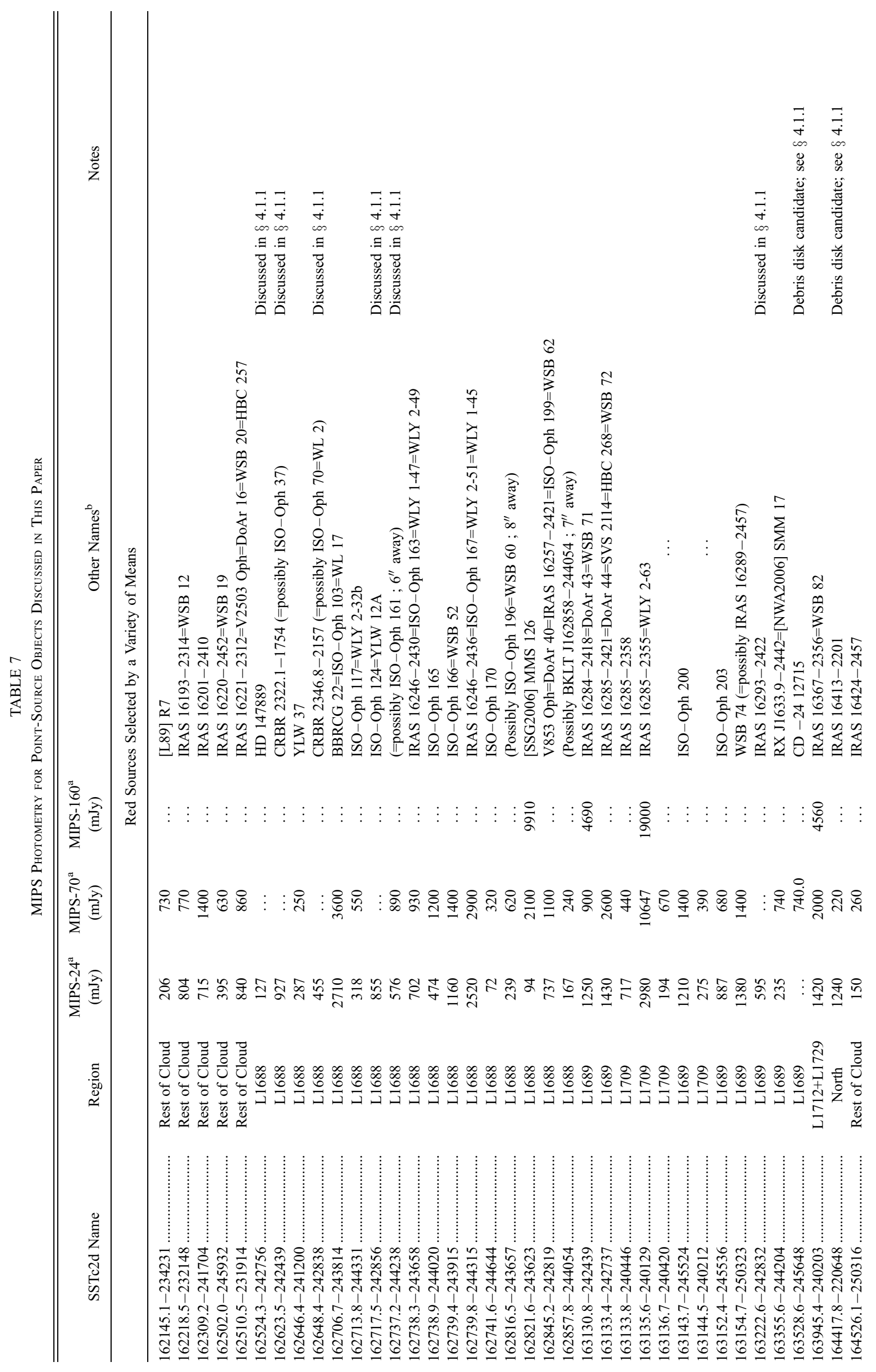




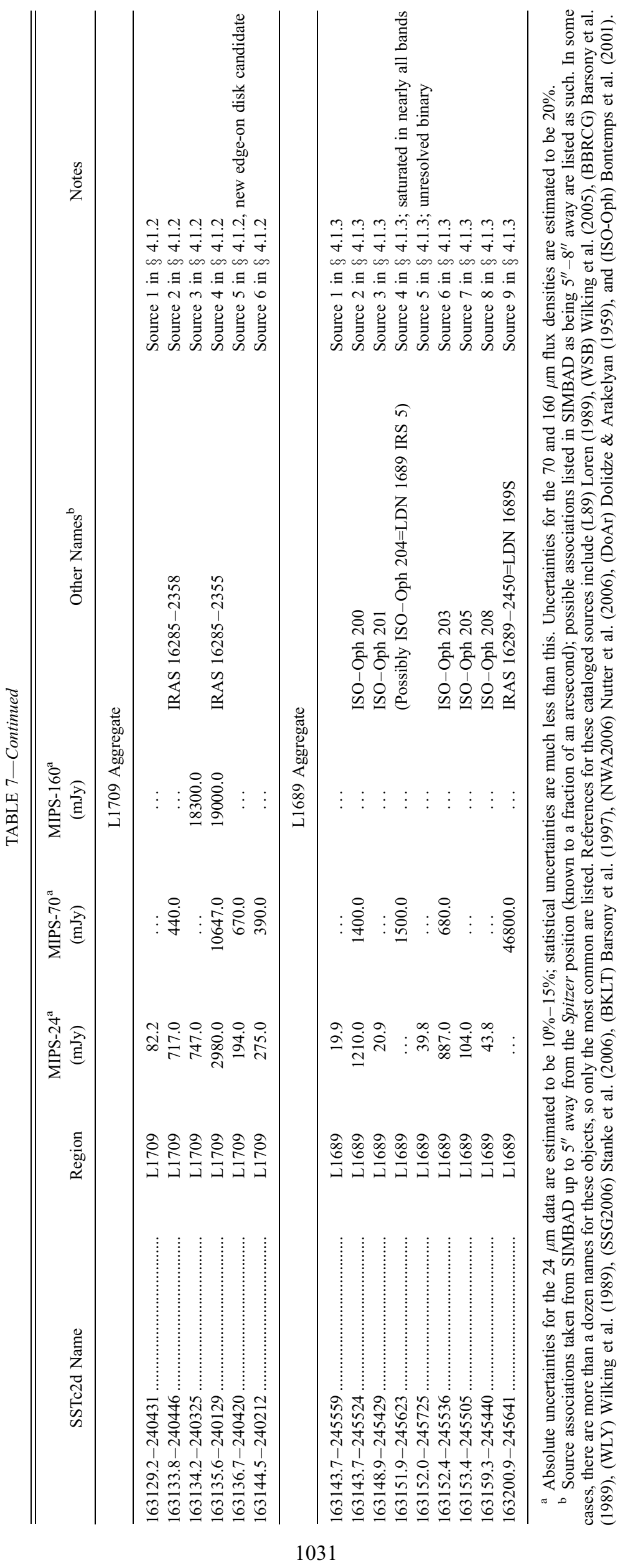




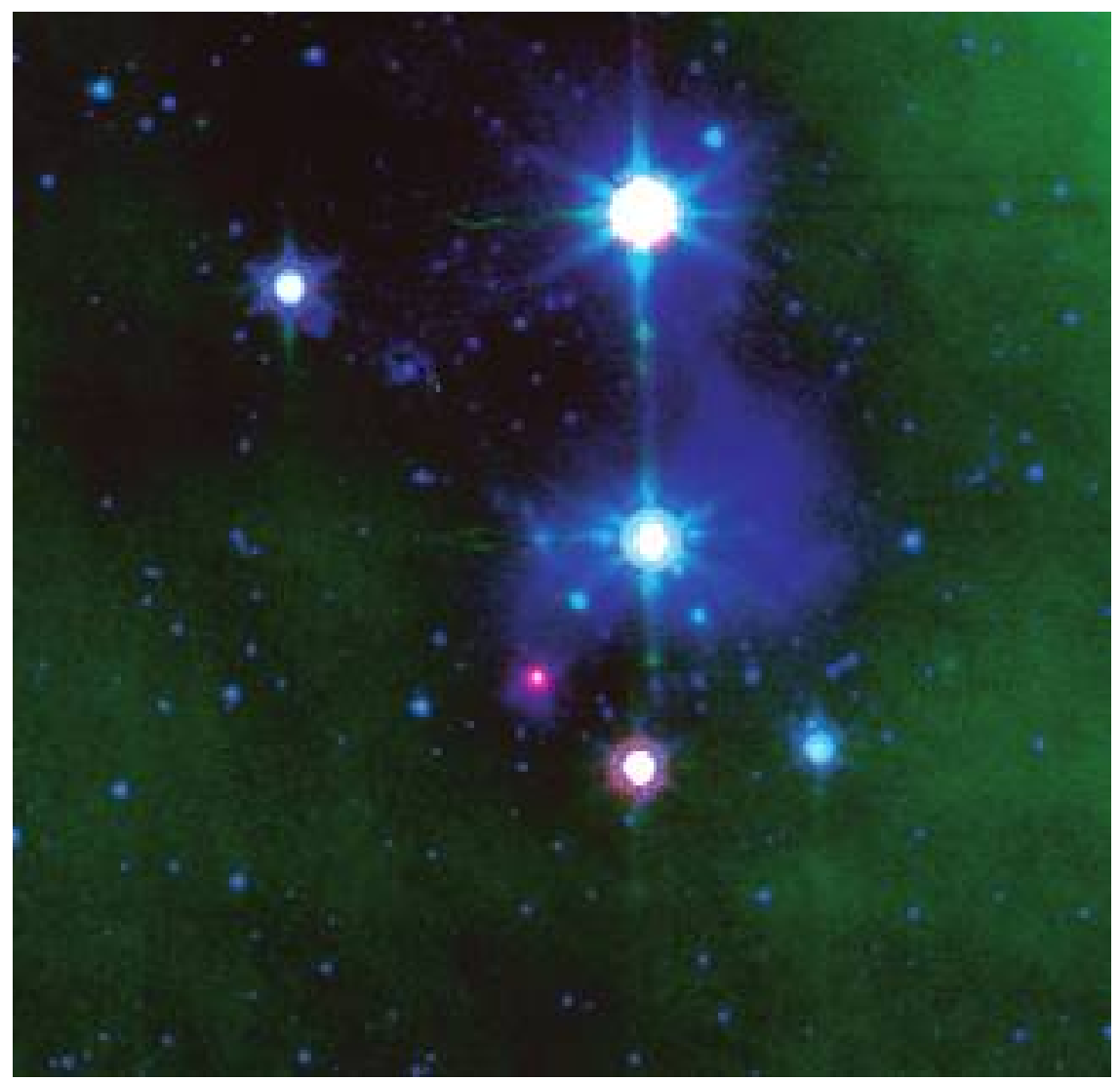

FIG. 18. - Three-color $9^{\prime} \times 9^{\prime}$ image of the L1709 aggregate. North is up, and east is left. The IRAC $4.5 \mu \mathrm{m}$ channel is shown in blue, IRAC $8 \mu \mathrm{m}$ in green, and MIPS $24 \mu \mathrm{m}$ in red. The candidate edge-on disk (source 5) is clearly extended in the $4.5 \mu \mathrm{m}$ image.

a few arcseconds away (Greene et al. 1994). Its SED is characteristic of an extincted stellar photosphere with an excess which appears to be present in the IRAC bands and extends to $24 \mu \mathrm{m}$. No $70 \mu \mathrm{m}$ source is detected. It lies close to another YSO (Oph S2; best seen in our $24 \mu \mathrm{m}$ image), and thus, there is potential SED confusion at longer wavelengths. Source SSTc2d 162648.4242838 is similar, although fainter. It also appears as a binary source in the IRAC $3.6 \mu \mathrm{m}$ band and can tentatively be identified with WL 2 (Wilking et al. 1989). Finally, SSTc2d 162737.2244238 (GY 301) is a faint stellar source with an almost flat spectrum out to $70 \mu \mathrm{m}$. All of these sources appear in high-extinction regions, and it appears likely that their extraordinary $K_{s}-[24]$ colors are primarily due to extinction at the $K_{s}$ band. Thus, these are likely normal flat-spectrum YSOs with disks seen at very high extinctions due to location within the cloud or envelope.

\subsubsection{The L1709 Aggregate and a Possible Edge-on Disk}

In L1709 there is a grouping of six bright $24 \mu \mathrm{m}$ point sources within $4.2^{\prime} \times 3.3^{\prime}$ (see Fig. 16). SEDs for all six of these objects appear in Figure 17, and MIPS fluxes for these objects appear in Table 7. As a shorthand, numbers between 1 and 6 appear in Figure 17; the corresponding formal c2d names appear in Table 7. IRAS 16285-2358 can be identified with source 2 in Figure 17. This object was selected as a candidate YSO by Beichman et al. (1986). IRAS 16285-2355, source 4 in Fig. 17, is also selected as one of the most extreme $K_{s}-[24]$ colors in the cloud. This well-known source (e.g., Visser et al. 2002 and references therein) shows a typical Class I or flat-spectrum SED rising through the mid-IR and flattening across the three MIPS bands. It appears to be a version with higher luminosity or more pole-on disk geometry of the same type of sources with extreme $K_{s}-[24]$ colors discussed in the previous section. Objects 1,3,5, and 6 in Figure 17 do not have any known counterparts in the literature. Source SSTc2d 163136.7-240420, source 5 in Figure 17, stands out for its similarity to edge-on disks models for objects like HH 30 (Burrows et al. 1996). The 2MASS+IRAC part of the SED resembles a normal stellar photosphere which peaks at $K$, albeit a very faint one. However, its 24 and $70 \mu \mathrm{m}$ fluxes are almost 2 orders of magnitude higher than the $8.0 \mu \mathrm{m}$ value. Edge-on disks such as HH 30 and HK Tau/c (Wood et al. 2002) can appear in scattered light until 10-20 $\mu \mathrm{m}$, suppressing both their short- and long-wavelength flux densities. A $3.6 \mu \mathrm{m}$ image is presented in Figure 18.

Five of the six sources in $\mathrm{L} 1709$ were observed in the $350 \mu \mathrm{m}$ continuum at the CSO. Two (L1709-3 and -4) were clearly detected and showed extended structure. For these sources, an elliptical aperture with radius of $1^{\prime}$ was used to calculate the flux density, excluding some of the low-level extended structure in the images. The L1709-5 (SSTc2d 163136.7) source is in a region of considerable structure; it appears to be sitting on a ridge of emission and shows a cometary structure extending toward the south-southeast. To the northwest $\left(\alpha=16^{\mathrm{h}} 31^{\mathrm{m}} 35.33^{\mathrm{s}}, \delta=\right.$ $-24^{\circ} 03^{\prime} 46.8^{\prime \prime}$ [J2000.0]) is another bowed extended ridge of emission, with a flux of $\sim 200 \mathrm{mJy}$. The CSO flux densities and upper limits are incorporated in the SEDs presented in Figure 17. We use the equations of Beckwith et al. (1990) to estimate lower limits on the circumstellar mass from these measurements. Presuming that the $350 \mu \mathrm{m}$ emission is optically thin and $T_{d}=$ $100 \mathrm{~K}, \kappa_{\nu}=0.071$, leading to lower limits on the circumstellar masses of $1.6 \times 10^{-2} M_{\odot}$ for SSTc2d 163135.6-240129 (L1709-4) and $6.4 \times 10^{-4} M_{\odot}$ for SSTc2d 163136.7-240420 (L1709-5). 


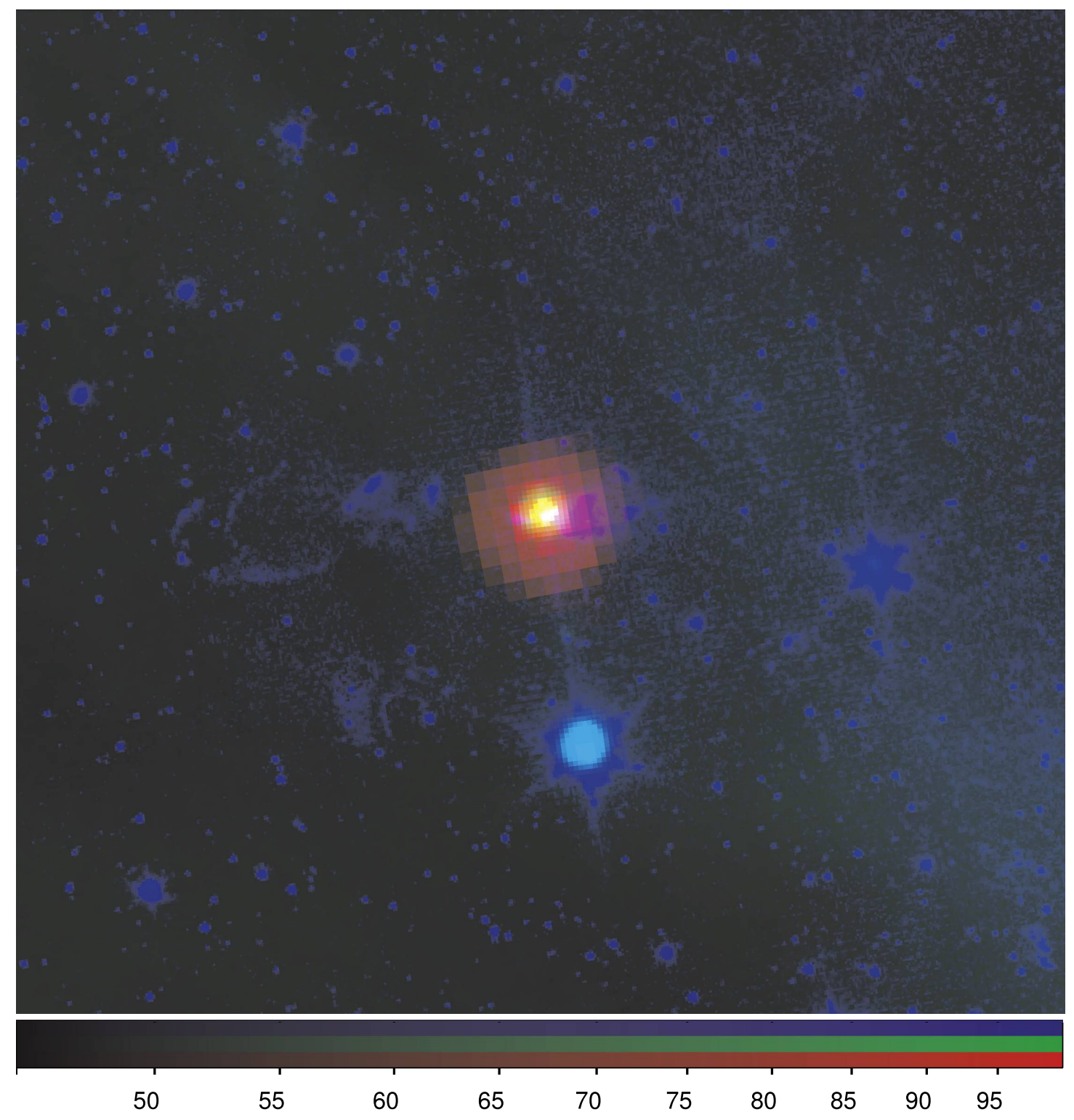

FIG. 19.-Three-color $9^{\prime} \times 9^{\prime}$ image of IRAS $16293-2422$. The IRAC $8 \mu \mathrm{m}$ channel is shown in blue, MIPS $24 \mu \mathrm{m}$ in green, and MIPS $70 \mu \mathrm{m}$ in red. The source is invisible at $8 \mu \mathrm{m}$, although the outflow emission and reflection nebulae are visible. Note especially the bow-shock features in blue to the left and right of the central source. In this image, north is up, and east is to the left. 

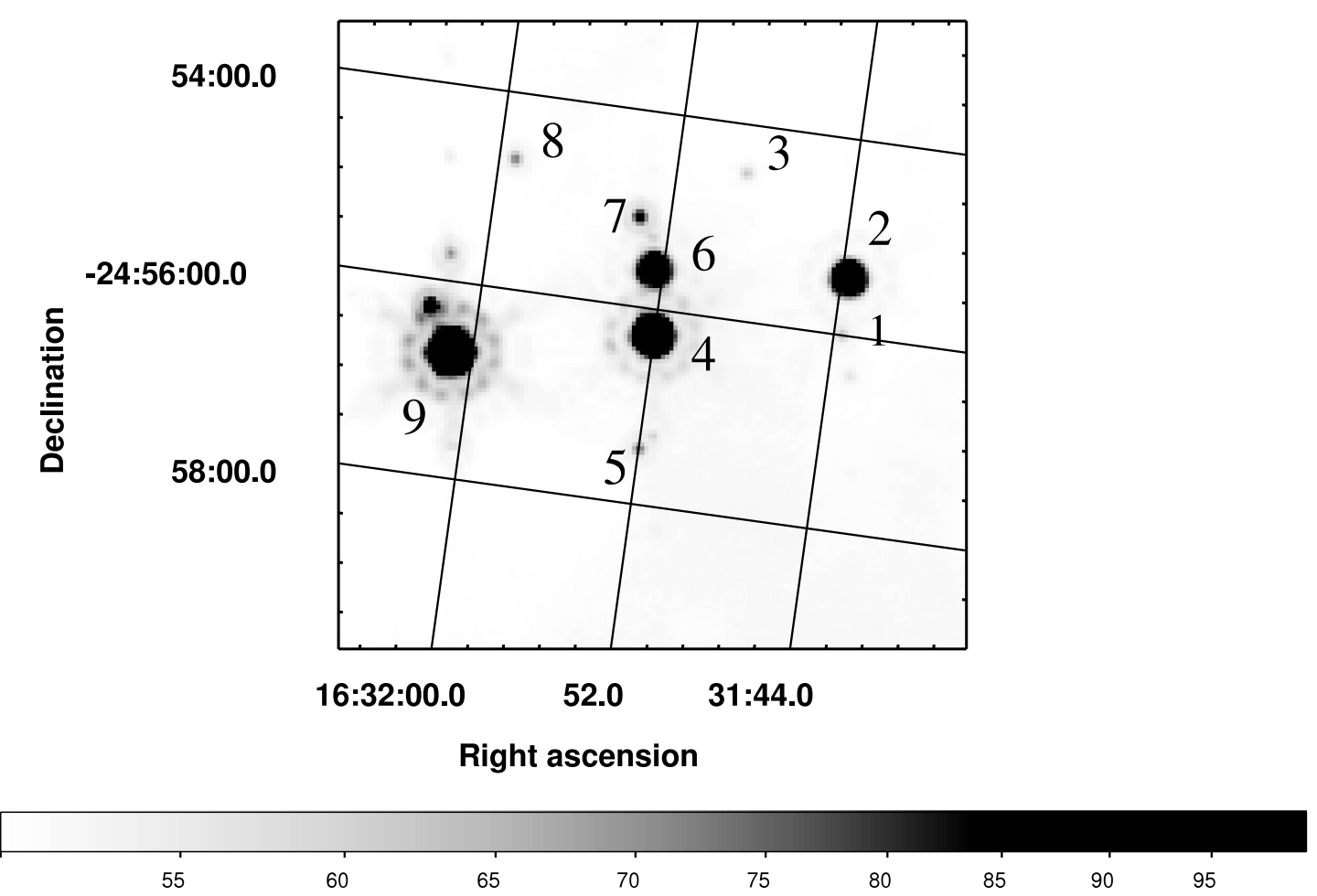

FIG. 20.-A $24 \mu \mathrm{m}$ image and finder chart for the L1689 aggregate.

\subsection{3. $L 1689$}

The famous Class 0 source IRAS 16293-2422 appears in the L1689 part of our MIPS maps. Interestingly, the source is entirely invisible in the IRAC $8 \mu \mathrm{m}$ image, although some of the jet and bow-shock emission from the outflow is plainly visible. The source itself begins to be seen at faint levels at $24 \mu \mathrm{m}$, is very bright at $70 \mu \mathrm{m}$, and is saturated at $160 \mu \mathrm{m}$. A color image is shown in Figure 19. The $8 \mu \mathrm{m}$ visibility of shocked gas very close to the invisible central source implies that the high-extinction region is very confined in areal coverage, suggesting an edge-on orientation to the disk/envelope system. This source has also been observed by the Spitzer Infrared Spectrograph (IRS; Jørgensen et al. 2005). In this work the authors interpret the rapid rise in the SED as a large inner hole in an extended envelope around a protobinary. We prefer to interpret the very large slope in the SED as an extinction effect from a highly inclined disk/envelope system.

There is an aggregate of nine stars bright at $24 \mu \mathrm{m}$ found clustered within a $5^{\prime}$ region within L1689. The MIPS-24 image of the aggregate is presented in Figure 20, and the Spitzer+2MASS SEDs for these stars appear in Figure 21. Several of these objects were also seen by $I S O$ and discussed as part of Bontemps et al. (2001).

Padgett et al. (2004) and Rebull et al. (2007) commented on small clusterings of sources bright at $24 \mu \mathrm{m}$ found in L1228 and Perseus, respectively. While Oph does not have such aggregates in as high abundance as Perseus, this grouping in L1689 is clearly a similar phenomenon in that there are several sources within $\sim 0.15 \mathrm{pc}$, all bright at $24 \mu \mathrm{m}$ and with a diversity of SEDs. As can be seen in Figure 21, some sources resemble reddened photospheres with just a hint of excess at $24 \mu \mathrm{m}$ (e.g., source 1), some appear to have Class II SEDs (e.g., source 8), and some have substantial excesses like Class I or flat-spectrum SEDs (e.g., source 6). Source 5 has a strangely shaped SED because it is an unresolved binary at $24 \mu \mathrm{m}$. Source 9 is so bright at $24 \mu \mathrm{m}$ that it leaves several latent images on the array (e.g., it is near saturation), so the unusual shape of the IRAC portion of the SED is likely to be a result of saturation in those bands. Object 4 is saturated at all Spitzer bands except for $70 \mu \mathrm{m}$. The proximity of these sources suggests that they are physically associated, despite their disparate SEDs. As in L1228 (Padgett et al. 2004), this aggregate seems to have sources at a variety of stages in their circumstellar evolution, although the sources must be nearly coeval.

\subsection{Clustering of YSOs in Ophiuchus}

Figure 1 shows the distribution of MIPS $24 \mu \mathrm{m}$ sources in the c $2 \mathrm{~d}$ maps. The sources appear to be randomly distributed in most of the map. However, as expected, there is a large overdensity of sources in the region around the Oph A core. Figure 22 accentuates this result, showing that relatively bright sources with $24 \mu \mathrm{m}$ excess are strongly concentrated in the core, as well as a scattering of small groups in L1689, northern L1688, and southern L1709. A modest number of excess sources are more widely distributed over the entirety of the L1719+1729 eastern filament, while Oph North has few bright $24 \mu \mathrm{m}$ excess sources. The "rest of the cloud," largely concentrated to the northeast of L1688, shows some intriguing small individual sources but no YSO aggregates.

Table 4 quantifies the degree of clustering seen by categorizing the objects with $24 \mu \mathrm{m}$ excess. Consideration of all $24 \mu \mathrm{m}$ sources with 2MASS associations shows that only 297 out of a total of 2590 sources are confined to the L1688 subregion. This would imply a large distributed population of potentially young sources in the cloud. However, when the selection criteria $K_{s}-$ [24] $>2$ and $K_{s}<14$ are applied, we see that more than half of the YSO candidates are confined to L1688. About $60 \%$ of the YSO candidates fall into the L1688, L1689, and L1709 regions. The clustering of sources with Class I and flat SEDs is more pronounced. A full $95 \%$ of these sources are confined to the central cluster and the L1709 and L1689 aggregates. In comparison, only $61 \%$ of the Class II candidates are located in these subregions. It is worth noting that the natures of the Class I and II candidates 


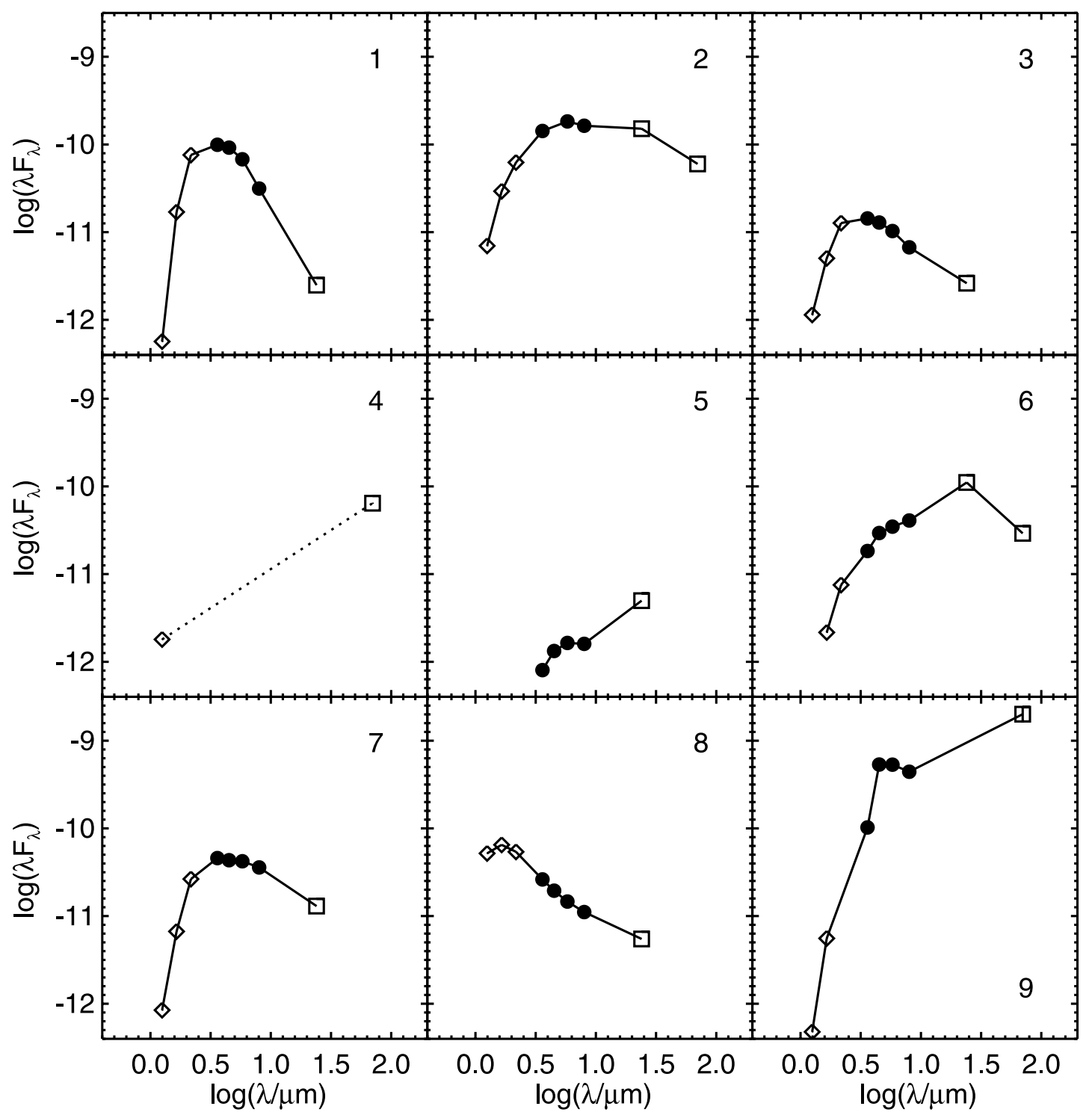

FIG. 21. - SEDs for nine YSO candidates bright at $24 \mu \mathrm{m}$ found tightly clumped together in L1689. Source 4 is saturated in all Spitzer bands except $70 \mu \mathrm{m}$. 


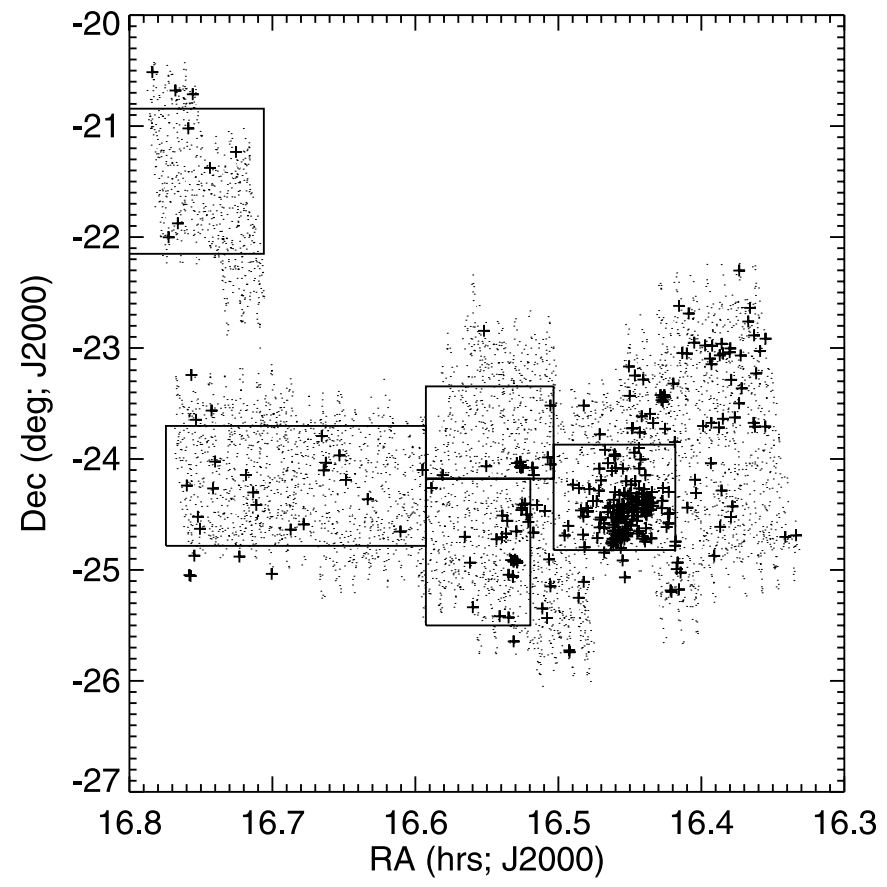

FIG. 22.-Locations of sources where $K_{s}-[24]>2$ and $K<14$.

remain unconfirmed by further study outside of the well-studied cluster and aggregates. Some percentage of these sources may well be extragalactic interlopers. Thus, star formation in the Ophiuchus clouds appears to be largely confined to the cluster and aggregates. Small aggregates of the type seen in Taurus, Lupus, and Perseus are found in the L1709 and L1689 regions, but not in the remainder of the filamentary clouds in Ophiuchus.

\section{CONCLUSIONS}

We have presented the $14.4 \mathrm{deg}^{2}$ c2d Spitzer MIPS longwavelength imaging survey of the Ophiuchus molecular cloud complex. Many point sources and a variety of extended emission structures on scales from circumstellar to intercloud are shown in our mosaicked images. Using point-source-fitting photometry, we have identified and measured the brightness of 5779 sources at $24 \mu \mathrm{m}, 81$ sources at $70 \mu \mathrm{m}$, and eight sources at $160 \mu \mathrm{m}$. Using criteria derived from a $K_{s}$ versus $K_{s}-$ [24] and other colorcolor and color-magnitude diagrams, we have identified some 323 candidate young stellar objects throughout the clouds. Several sources with extreme colors are discussed, and a candidate edge-on disk is identified. Our survey suggests that although the numbers of forming stars are dominated by the L1688 cluster and small aggregates in L1689 and L1709, there may be a population of Class II sources lurking in the more tenuous parts of the cloud. This distributed population could contain up to $40 \%$ of the YSO candidates in Ophiuchus.

Most of the support for this work, part of the Spitzer Space Telescope Legacy Science Program, was provided by NASA through contracts 1224608,1230782 , and 1230779 , issued by the Jet Propulsion Laboratory, California Institute of Technology, under NASA contract 1407 . We thank the Lorentz Center in Leiden for hosting several meetings that contributed to this paper. Support for J. K. J. and P. C. M. was provided in part by a NASA Origins grant, NAG5-13050. Astrochemistry in Leiden is supported by a NWO Spinoza grant and a NOVA grant. K. E. Y. was supported by NASA under grant NGT5-50401 issued through the Office of Space Science. This research has made use of NASA's Astrophysics Data System (ADS) Abstract Service, and of the SIMBAD database, operated at CDS, Strasbourg, France. This research has made use of data products from the Two Micron All Sky Survey, which is a joint project of the University of Massachusetts and the Infrared Processing and Analysis Center, funded by the National Aeronautics and Space Administration and the National Science Foundation. These data were served by the NASA/IPAC Infrared Science Archive, which is operated by the Jet Propulsion Laboratory, California Institute of Technology, under contract with the National Aeronautics and Space Administration. The research described in this paper was partially carried out at the Jet Propulsion Laboratory, California Institute of Technology, under contract with the National Aeronautics and Space Administration.

\section{REFERENCES}

Allen, L. E., Myers, P. C., Di Francesco, J., Mathieu, R., Chen, H., \& Young, E. 2002, ApJ, 566, 993

Barsony, M., Carlstrom, J. E., Burton, M. G., Russell, A. P. G., \& Garden, R. 1989, ApJ, 346, L93

Barsony, M., Kenyon, S., Lada, E. A., \& Teuben, P. J. 1997, ApJS, 112, 109

Barsony, M., Ressler, M. E., \& Marsh, K. A. 2005, ApJ, 630, 381

Beckwith, S. V. W., Sargent, A. I., Chini, R. S., \& Guesten, R. 1990, AJ, 99, 924

Beichman, C. A., et al. 1986, ApJ, 307, 337

. 1988, IRAS Catalogs and Atlases, Vol. 1 (Pasadena: Caltech)

Bontemps, S., et al. 1998, in ASP Conf. Ser. 132, Star Formation with the Infrared Space Observatory, ed. J. Yun \& R. Liseau (San Francisco: ASP), 141

- 2001, A\&A, 372, 173

Bouvier, J., \& Appenzeller, I. 1992, A\&AS, 92, 481

Burrows, C. J., et al. 1996, ApJ, 473, 437

Chapman, N., et al. 2007, ApJ, 667, 288

Chini, R. 1981, A\&A, 99, 346

Comeron, F., Rieke, G. H., Burrows, A., \& Rieke, M. J. 1993, ApJ, 416, 185

Cushing, M. C., Tokunaga, A. T., \& Kobayashi, N. 2000, AJ, 119, 3019

Devito, B., \& Hayward, T. L. 1998, ApJ, 504, L43

de Geus, E. J., de Zeeuw, P. T., \& Lub, J. 1989, A\&A, 216, 44

de Zeeuw, P. T., Hoogerwerf, R., de Bruijne, J. H. J., Brown, A. G. A., \& Blaauw, A. 1999, AJ, 117, 354

Dolidze, M., \& Arakelyan, M. 1959, Astron. Zh., 36, 444

Duchêne, G., Bouvier, J., Bontemps, S., André, P., \& Motte, F. 2004, A\&A, 427, 651

Evans, N. J., II, et al. 2003, PASP, 115, 965

Flaherty, K. M., Pipher, J. L., Megeath, S. T., Winston, E. M., Gutermuth, R. A., Muzerolle, J., Allen, L. E., \& Fazio, G. G. 2007, ApJ, 663, 1069 Gomez, M., Whitney, B. A., \& Wood, K. 1998, AJ, 115, 2018

Gorlova, N., et al. 2004, ApJS, 154, 448

Grasdalen, G. L., Strom, S. E., \& Strom, K. M. 1973, ApJ, 184, L53

Greene, T. P., \& Meyer, M. R. 1995, ApJ, 450, 233

Greene, T. P., Wilking, B. A., André, P., Young, E. T., \& Lada, C. J. 1994, ApJ, 434,614

Greene, T. P., \& Young, E. T. 1992, ApJ, 395, 516

Guglielmo, F., Epchtein, N., Le Bertre, T., Fouque, P., Hron, J., Kerschbaum, F., \& Lepine, J. R. D. 1993, A\&AS, 99, 31

Haisch, K. E., Jr., Barsony, M., Greene, T. P., \& Ressler, M. E. 2002, AJ, 124, 2841

Harvey, P. M., et al. 2006, ApJ, 644, 307

2007, ApJ, 663, 1139

Jørgensen, J. K., et al. 2005, ApJ, 631, L77

. 2006, ApJ, 645, 1246

Klose, S. 1986, Ap\&SS, 128, 135

Lada, C. J., \& Wilking, B. A. 1984, ApJ, 287, 610

Loren, R. B. 1989, ApJ, 338, 902

Luhman, K. L., \& Rieke, G. H. 1999, ApJ, 525, 440

Makovoz, D., Roby, T., Khan, I., \& Hartley, B. 2006, Proc. SPIE, 6274, 10

Martín, E. L., Montmerle, T., Gregorio-Hetem, J., \& Casanova, S. 1998, MNRAS, 300, 733

Moshir, M., Kopman, G., \& Conrow, T. 1992, IRAS Faint Source Survey, Explanatory Supplement, Ver. 2 (Pasadena: Caltech) 
Natta, A., Testi, L., \& Randich, S. 2006, A\&A, 452, 245

Nutter, D., Ward-Thompson, D., \& André, P. 2006, MNRAS, 368, 1833

Padgett, D. L., et al. 2004, ApJS, 154, 433 ApJ, 645, 1283

Phelps, R. L., \& Barsony, M. 2004, AJ, 127, 420

Porras, A., et al. 2007, ApJ, 656, 493

Rebull, L., et al. 2007, ApJS, 171, 447

Ressler, M., \& Barsony, M. 2003, ApJ, 584, 832

Ridge, N., et al. 2006, AJ, 131, 292

Rieke, G. H., Ashok, N. M., \& Boyle, R. P. 1989, ApJ, 339, L71

Robitaille, T. P., Whitney, B. A., Indebetouw, R., Wood, K., \& Denzmore, P. 2006, ApJS, 167, 256

Shupe, D., et al. 2008, ApJ, in press

Stanke, et al. 2006, A\&A, 447, 609
Stapelfeldt, K. R., et al. 2006, BAAS Planet. Sci. Meet. Abs., 38, 58.06

Strom, K. M., Kepner, J., \& Strom, S. E. 1995, ApJ, 438, 813

Visser, A. E., Richer, J. S., \& Chandler, C. J. 2002, AJ, 124, 2756

Vrba, F. J., Strom, K. M., Strom, S. E., \& Grasdalen, G. L. 1975, ApJ, 197, 77

Wainscoat, R. J., \& Cowie, L. L. 1992, AJ, 103, 332

Wilking, B. A., Greene, T. P., \& Meyer, M. R. 1999, AJ, 117, 469

Wilking, B. A., \& Lada, C. J. 1983, ApJ, 274, 698

Wilking, B. A., Lada, C. J., \& Young, E. T. 1989, ApJ, 340, 823

Wilking, B. A., Meyer, M. R., Robinson, J. G., \& Greene, T. P. 2005, AJ, 130, 1733

Wood, K., Lada, C. J., Bjorkman, J. E., Kenyon, S. J., Whitney, B., \& Wolff, M. J. 2002, ApJ, 567, 1183

Young, K. E., et al. 2005, ApJ, 628, 283

2006, ApJ, 644, 326 